\title{
Instabilidade Detectável por Jatos de Pontos de Equilíbrio de Sistemas Lagrangeanos Conservativos
}

Fábio Armando Tal

Orientador: Manuel Valentim de Pera Garcia

\begin{abstract}
Tese apresentada para obtenção do título de Doutor em Matemática Aplicada pelo Departamento de Matemática Aplicada do Instituto de Matemática e Estatística da Universidade de São Paulo.
\end{abstract}

Durante a preparação deste trabalho, o autor recebeu bolsa da CAPES. 
Aos meus pais, Mendy e Lica, e à minha esposa, Thaís. 


\section{Agradecimentos}

Agradeço primeiramente ao professor Manuel Garcia, não só pela excelente orientação em todo o processo de confecção desta tese, e pelos anos de paciência e dedicação à minha formação como matemático, mas principalmente pela grande amizade e por ter me mostrado toda a beleza e o prazer que existe em fazer matemática. Ele é, indubitavelmente, o principal "culpado" pela existência desta tese.

Agradeço aos professores Angêlo Barone-Netto, Sônia Regina Leite Garcia, Cláudio Possani e Paulo José da Silva e Silva, pela paciência em examinar meus resultados e pelas sugestões que em muito me auxiliaram a encontrar a direção correta para prosseguir.

Agradeço aoś meus pais, sou quem sou por obra deles, e agradeço ao meu irmã̃o; André, por todo o amor que me dedicou.

Agradeço à minha esposa, Thaís, pela paciência com os meus humores, pelo carinho e incentivo que muito me motivou, não só a realizar este trabalho, mas em todos os outros aspectos da vida.

Agradeço à Laura, ao Axel e ao Álvaro, vocês fizeram a minha vida mais divertida e significativa.

Finalmente, agradeço aos professores Clodoaldo Ragazzo, Henrique Dreifus, Sérgio Oliva e Carlos Humes que me auxiliaram nos momentos difíceis. 
Resumo

Neste trabalho estudamos a estabilidade segundo Liapounof de pontos de equilíbrio de sistemas lagrangeanos conservativos do tipo $L=T-\pi$, onde $T$ é a energia cinética e $\pi$ é a energia potencial com um ponto crítico na origem.

Nosso principal resultado fornece, para lagrangeanos com dois graus de liberdade, uma condição necessária e suficiente para que o jato de ordem $k$ de $\pi$ na origem assegure a instabilidade do equilíbrio $(\mathbf{0} ; \mathbf{0})$ das equações do movimento. Esta instabilidade é decorrente da existência de uma trajetória assintótica à origem no passado. Um corolário deste resultado é a existência desta trajetória assintótica para potenciais analíticos sem mínimo local na origem, uma questão que estava em aberto até o presente momento.

$\mathrm{Na}$ tese também apresentamos um exemplo de dois sistemas lagrangeanos, $L_{1}$ e $L_{2}$, com a mesma energia potencial polinomial e mostramos que, mesmo localmente, os fluxos das equações do movimento destes dois sistemas não são conjugados, sendo que a variedade instável do equilíbrio do primeiro sistema tem dimensão 2 , enquanto que no segundo sistema esta variedade tem dimensão 1. Isto evidencia o papel da energia cinética no estudo dos pontos de equilíbrio de sistemas conservativos.

Finalmente, provamos também alguns resultados sobre como encontrar trajetórias assintóticas para equilíbrios de sistemas de equações diferenciais ordinárias utilizando funções auxiliares, aplicando esta técnica para sistemas bi e tri-dimensionais. 


\begin{abstract}
In this work we study the stability according to Liapounof of equilibria of conservative Lagrangian systems of the type $L=$ $T-\pi$, where $T$ is the kinetic energy and $\pi$ is the potential energy with a critical point at the origin.

Our main result provides, for Lagrangians with two degrees of freedom, a necessary and sufficient condition for the $k$ jet of $\pi$ at the origin ensures the instability of the equilibrium $(\mathbf{0} ; \mathbf{0})$. This instability is achieved by means of an asymptotic trajectory to the origin in the past. An corollary is that such trajectory exists for Lagrangians with analytic potentials that do not have a minimum at the origin, an open question until now.

In the thesis we also provide an example of two Lagrangian systems, $L_{1}$ and $L_{2}$, with the same polynomial potential energy, and we show that the associated flow are not locally equivalent, since the unstable manifold for the first system has dimension 2, while the unstable manifold for the second system has dimension 1. This underlines the role played by the kinetic energy in the study of equilibria of conservative systems.

Also, we prove some results on how to use auxiliary functions to find solutions asymptotic to an equilibrium of systems of differential equations, and we apply this technique for two and tridimensional systems.
\end{abstract}


Índice

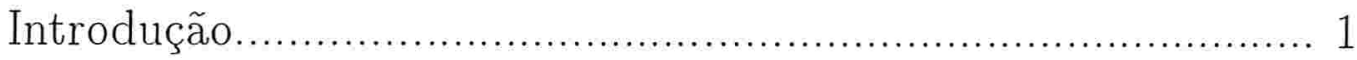

Trajetórias Assintóticas Resistentes a Perturbações ................. 11

A Inversão do Teorema de Dirichlet-Lagrange.......................... 47

Influência da Energia Cinética na Estab. do Equil. ................. 66

K-Decidibilidade e Curva de Mínimos Verticais......................... 72

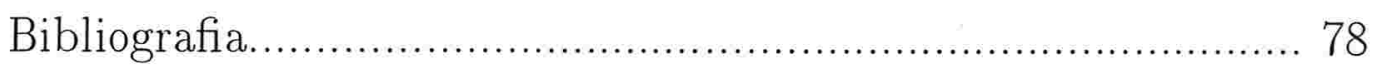




\section{Capítulo 1 Introdução}

\subsection{Colocando o Problema}

A principal motivação para esta tese é o estudo da estabilidade Liapounof de pontos de equilíbrio de sistemas lagrangeanos conservativos. Por sistema lagrangeano entenderemos uma função $L: \Omega \times \mathbf{R}^{n} \rightarrow \mathbf{R}$ de classe $\mathcal{C}^{k}$, onde $k \geq 2$ e $\Omega$ é um aberto contido em $\mathbf{R}^{n}$, e um sistema de equações diferenciais de segunda ordem

$$
\frac{d}{d t} \frac{\partial L}{\partial \dot{q}}-\frac{\partial L}{\partial q}=0
$$

onde $\frac{\partial^{2} L}{\partial \dot{q}^{2}}$ tem posto máximo.

De maneira geral, a menos que se explicite o contrário, consideraremos lagrageanos da forma $L=T-\pi$, onde $T: \Omega \times$ $\mathbf{R}^{n} \rightarrow \mathbf{R}$ de classe $\mathcal{C}^{k}$ é uma forma quadrática definida positiva nas velocidades, chamada energia cinética; $\pi: \Omega \subset \mathbf{R}^{n} \rightarrow \mathrm{R} \in$ $\mathcal{C}^{k}$, chamada energia potencial e $\Omega$ é uma vizinhança aberta da origem.

Para lagrangeanos como os considerados, as equações do movimento ficam na forma

$$
\frac{d}{d t} \frac{\partial T}{\partial \dot{q}}-\frac{\partial T}{\partial q}=-\nabla \pi
$$


É fato conhecido que os únicos pontos de equilíbrio das equações acima são do tipo $(q, \dot{q})=\left(q_{0}, 0\right)$, onde $q_{0}$ é um ponto crítico da energia potencial. Neste trabalho admitiremos que $\pi(0)=\|\nabla \pi(0)\|=0$ é um ponto crítico da energia potencial. O termo "origem" será utilizado indiscriminadamente para representar tanto $\mathbf{0} \in \mathbf{R}^{n}$ quanto $(\mathbf{0}, \mathbf{0}) \in \mathbf{R}^{n} \times \mathbf{R}^{n}$, mas veremos que o contexto não permitirá confusões.

Também vale notar que, quando mencionarmos "a estabilidade de um equilíbrio", estaremos nos referindo à estabilidade de Liapounof do mesmo.

\subsection{Breve Histórico do Problema}

O estudo da estabilidade dos pontos de equilíbrio da equação (1.1) acima começou no século XVIII, acumulando, até os dias de hoje, uma extensa literatura. O principal resultado histórico que deu início a este ramo de pesquisa foi enunciado por Lagrange em 1788 e demonstrado por Dirichlet em 1846.

Teorema 1.1 (Dirichlet-Lagrange) Suponha que $\pi(q)>0$, para todo $q \in \Omega \backslash 0$. Então a origem é um equilíbrio estável segundo Liapounof das equações (1.1).

Após a demonstração deste teorema, vários matemáticos buscaram provar a recíproca do mesmo, isto é, que se $\pi$ não tivesse um mínimo estrito na origem, então o equilíbrio seria instável. Um contra-exemplo para este fato foi fornecido, em 1904, por Painlevé que mostrou, para a energia potencial

$$
\pi(q)=\left\{\begin{array}{c}
e^{-\frac{1}{q^{2}}} \sin (q), \text { se } q \neq 0 \\
0, \text { se } q=0
\end{array}\right.
$$


a estabilidade da origem, independente da energia cinética considerada.

Criou-se então um campo de estudo, apelidado pela literatura de "Inversão do Teorema de Dirichlet-Lagrange". Passou-se a buscar condições sob as quais se pudesse garantir a estabilidade ou instabilidade da origem.

Um dos resultados mais antigos nesta direção e que merece ser mencionado foi o de Liapounof, que provou

Teorema 1.2 (Liapounof-1897) Suponha que $\pi=\pi_{2}+R$ onde $\pi_{2}$ é um polinômio homogêneo de grau 2 sem minimo na origem e $R=o\left(\|q\|^{2}\right)$. Então a origem é instável.

É interessante notar que a condição imposta sobre a energia potencial garante que a mesma não tem mínimo na origem.

Depois deste seguiram os teoremas:

Teorema 1.3 (Cetaev - 1952) Suponha que $\pi$ é um polinômio homogêneo, e que este polinômio não tem minimo estrito na origem. Então a origem é instável.

Teorema 1.4 (Hagedorn-1971) Suponha que $\pi(q)<0$ em $\Omega \backslash\{0\}$ (0 é um ponto de máximo estrito local). Então a origem é instável.

Teorema 1.5 (Palamodov-1978; Tagliaferro-1980) Suponha que $q \in \mathbf{R}^{2}, \pi \in \mathcal{C}^{w}, 0$ não é um ponto de minimo de $\pi$ e $T(q, \dot{q})=$ $\frac{\dot{q}_{1}^{2}+\dot{q}_{2}^{2}}{2}$. Então a origem é instável.

Teorema 1.6 (Tagliaferro-1980)Suponha que $\pi(q) \leq 0$ em $\Omega \backslash\{0\}$ (0 é um ponto de máximo local, estrito ou não). Então a origem é instável. 
Na época, ainda resistia a seguinte conjectura, não desqualificada pelo exemplo de Painlevé:

Conjectura 1.1 A origem é um equilibrio estável das equações (1.1) se, e somente se, a componente conexa do conjunto

$$
A=\{q \mid \pi(q) \leq 0\}
$$

que contém a origem for $\{\mathbf{0}\}$.

Esta conjectura é verdadeira para sistemas com um grau de liberdade e portanto, neste contexto, a recíproca do teorema de Dirichlet-Lagrange foi conseguida. Porém, para sistemas com dois ou mais graus de liberdade, o seguinte exemplo de Laloy(1975) mostrou ser falsa a conjectura:

Exemplo 1.1 Sejam

$$
\begin{aligned}
& \pi\left(q_{1}, q_{2}\right)=\left\{\begin{array}{c}
e^{-\frac{1}{q_{1}^{2}}} \sin \left(\frac{1}{q_{1}}\right)-e^{-\frac{1}{q_{2}^{2}}}\left(\sin \left(\frac{1}{q_{2}}\right)+q_{2}^{2}\right), \text { se } q_{1} \cdot q_{2} \neq 0, \\
e^{-\frac{1}{q_{1}^{2}}} \sin \left(\frac{1}{q_{1}}\right), \text { se } q_{1} \neq 0, q_{2}=0, \\
-e^{-\frac{1}{q_{2}^{2}}}\left(\sin \left(\frac{1}{q_{2}}\right)+q_{2}^{2}\right), \text { se } q_{1}=0, q_{2} \neq 0,
\end{array}\right. \\
& T\left(q_{1}, q_{2}, \dot{q}_{1}, \dot{q}_{2}\right)=\frac{\dot{q}_{1}^{2}+\dot{q}_{2}^{2}}{2}, \quad 0, \text { se } q_{1}^{2}+q_{2}^{2}=0,
\end{aligned}
$$

então a origem é um equilíbrio estável de (1.1).

Veja que com esta energia cinética podemos escrever o nosso sistema lagrangeano como soma de dois sistemas lagrangeanos com um grau de liberdade. É digno de nota que, pela primeira vez nos trabalhos citados, a energia cinética parece ter alguma importância no estudo da estabilidade. Note que a estabilidade só foi demonstrada para uma energia cinética particular, ao contrário dos resultados passados que em nenhum instante supuseram algo 
sobre a energia cinética, a não ser uma certa regularidade. É uma questão em aberto analisar a estabilidade desta energia potencial, quando a energia cinética não permite tal simplificação do sistema.

Posteriormente, Laloy e Pfeiffer demonstraram que:

Teorema 1.7 (Laloy-Pfeiffer-1982) Suponha que $q \in \mathbf{R}^{2}, \pi \in$ $\mathcal{C}^{\omega}, \pi$ tem minimo estritamente brando (i.e. $\pi(q) \geq 0$ e existe $\left(q_{k}\right)$ sequência de números reais, $\lim _{k \rightarrow \infty} q_{k}=0$, e $\pi\left(q_{k}\right)=0$ ). Então a origem é um equilíbrio instável.

Este é o principal resultado, até o presente momento, sobre a instabilidade de energias potenciais com mínimos não estritos na origem. Pouco se sabe sobre a estabilidade de potenciais com mínimo estritamente brando para sistemas com mais graus de liberdade, mesmo que a energia potencial seja analítica.

Posteriormente, no fim da década de oitenta e início da década de noventa, foram provados alguns resultados ligando a estabilidade de $(0,0)$ com a análise dos jatos da energia potencial na origem:

Teorema 1.8 (Moauro-Negrini-1989) Suponha que $k \geq 2, \pi(q) \in$ $\mathcal{C}^{k+3}(\Omega, \mathbf{R}), \pi=\pi_{k}+W$, onde $\pi_{k}$ é um polinômio homogêneo de grau $k$, sem minimo na origem, e $W=o\left(\|q\|^{k}\right)$. Então a origem é um equilíbrio instável, e existe uma solução de (1.1) assintótica à origem.

Teorema 1.9 ((Maffei-Moauro-Negrini-1991) Suponha que $k \geq$ $2, \pi\left(q_{1}, q_{2}\right) \in \mathcal{C}^{k+3}\left(\Omega_{1} \times \Omega_{2} \subset \mathbf{R}^{n} \times \mathbf{R}^{m}, \mathbf{R}\right)$,

$\pi\left(q_{1}, q_{2}\right)=\pi_{2}\left(q_{1}\right)+\pi_{k}\left(q_{2}\right)+W\left(q_{2}\right)$, onde $\pi_{2}$ é uma forma quadrática definida positiva, $\pi_{k}$ é um polinômio homogêneo de grau $k$, sem minimo na origem, e $W=o\left(\left\|q_{2}\right\|^{k}\right)$. Então a origem é um equilíbrio instável, e existe uma solução de (1.1) assintótica à origem. 
Teorema 1.10 (Garcia, M.-1992) Suponha que $q \in \mathbf{R}^{2}, \pi=\pi_{s}+$ $\cdots+\pi_{r}+\pi_{r+1}+\cdots+\pi_{m}+W$, onde $\pi_{j}$ é um polinômio homogêneo de grau $j, W=o\left(|q|^{m}\right), \pi_{j}(q) \geq 0, j \in[s ; r], \pi_{j}(q) \leq 0, j \in$ $[r+1 ; m]$ e 0 não é um ponto de mínimo de $\sum_{j=s}^{m} \pi_{j}$. Então a origem é um equilíbrio instável.

Por último, em 1995 Palamodov provou um excelente resultado para potenciais analíticos:

Teorema 1.11 (Palamodov-1995) Suponha que $\pi \in \mathcal{C}^{\omega}$, e que $\pi$ não tem minimo na origem. Então a origem é um equilíbrio instável.

Vários outros resultados seriam dignos de nota, porém não seguem a linha principal que pretendemos tratar nesta tese, a saber, o estudo da estabilidade ou instabilidade da origem através da análise do jato de ordem $k$ da energia potencial $\pi$ na origem.

Outras linhas que têm despertado o interesse de alguns pesquisadores são: o estudo da estabilidade segundo Liapounof de lagrangeanos com forças giroscópicas (o chamado Problema de Rouche), e o estudo de lagrangeanos sujeitos a forças não-conservativas. O Leitor interessado poderá consultar os excelentes surveys de Negrini (ver $[\mathrm{N}]$ ) e de Rumyantsev e Sosnitski (ver $[\mathrm{RS}])$.

\subsection{Nosso Trabalho}

Uma breve análise dos resultados citados nos levaria a observar que, nos teoremas 1.2, 1.8, 1.9 e 1.10, a energia potencial é do tipo $\pi=P+R$, onde $P$ é o jato k de $\pi$ e $R=o\left(\|q\|^{k}\right)$. Além disso, pode-se provar, a partir das hipóteses apenas sobre $P$, que a energia potencial não tem mínimo na origem. 
Uma conjectura muito natural buscando estender estes resultados, que parece ter sido sugerida de forma informal pelo próprio Liapounof no início do século passado, foi posteriormente proposta por Ângelo Barone-Netto, a saber:

Conjectura 1.2 Seja $\pi=P+R$ uma energia potencial onde $P=j^{k} \pi$ e $R=o\left(\|q\|^{k}\right)$. Se apenas da análise de $P$ for possivel afirmar que $\pi$ não tem minimo em $\mathbf{0}$, então a origem é um equilíbrio instável.

A frase "Se apenas da análise de $P$ for possível afirmar que $\pi$ não tem mínimo em 0 " carece de uma definição mais precisa, a qual foi dada por Ângelo Barone-Netto em sua tese de livre-docência, através do conceito mais amplo de k-decidibilidade (Definição 5.1 do Apêndice).

Posteriormente foi mostrado em [BGZ] que, para qualquer função analítica $f$ que não tenha mínimo na origem, existe um $k \in \mathbf{N}$ tal que $j^{k} f$ mostra que a mesma não tem mínimo. Logo, uma prova da conjectura anterior seria em particular uma extensão do resultado de Palamodov.

O resultado central desta tese demonstra a conjectura no caso particular de dois graus de liberdade, e mostra ainda que existe uma trajetória assintótica para a origem. Vale notar que, mesmo para funções analíticas, a existência de tal trajetória não era assegurada, e Kozlov (ver [Koz]) menciona esta questão explicitamente.

Para obtermos o resultado central, também foi necessário um estudo um pouco mais aprofundado de k-decidibilidade no contexto bidimensional. Um resultado inédito, essencial na demonstração do teorema central, que surgiu neste estudo aparece no Apêndice. 
O Capítulo 3 demonstra o teorema central da tese.

Para demonstrarmos o teorema, utilizamos uma técnica geométrica valendo-nos de funções auxiliares para construirmos regiões aderentes à origem onde estudamos o comportamento do campo no bordo destas regiões tentando detectar condições suficientes para garantir a existência de trajetórias assintóticas.

No processo de desenvolvimento desta técnica outros resultados particulares apareceram, os quais, na nossa opinião, têm relevância própria, não servindo apenas para ilustrar a técnica. Estes resultados estão descritos no Capítulo 2, que não trata de sistemas lagrangeanos e sim da persistência de trajetórias assintóticas para sistemas de equações diferenciais ordinárias de primeira ordem que são fracamente perturbados.

Neste Capítulo descrevemos quando uma trajetória assintótica particular, tangente a uma direção dada, de um campo do tipo

$$
\dot{X}=P(X),
$$

resiste a perturbações do tipo

$$
\dot{X}=P(X)+R(X)
$$

onde $R$ tem ordem maior do que $P$ numa região "suficientemente tangente" à trajetória dada na origem. O Capítulo se restringe aos casos bi e tridimensionais, exceto por alguns teoremas gerais de existência de trajetórias assintóticas por meio de funções auxiliares.

Resta ainda lembrar uma questão abordada na introdução histórica, mas que na nossa opinião ainda não recebeu a devida atenção na literatura. Nota-se que, dentre todos os resultados mencionados na introdução histórica, apenas o exemplo de Laloy faz alguma restrição à energia cinética envolvida e, mesmo para 
este exemplo, não se sabe o que aconteceria caso a energia cinética fosse outra. Qual é o papel da energia cinética na estabilidade de um ponto de equilíbrio? Existe alguma energia potencial $\pi$ e um par de energias cinéticas $T_{1}$ e $T_{2}$ tais que o equilíbrio do lagrangeano $L_{1}=T_{1}+\pi$ é estável e o equilíbrio do lagrangeano $L_{2}=T_{2}+\pi$ é instável?

O único resultado que conhecemos nesta direção é o de Garcia, S.( ver $[$ Gar - S]), que dá uma resposta afirmativa a esta questão no contexto de sistemas lagrangeanos não conservativos.

O último capítulo desta tese mostra um exemplo em dois graus de liberdade, onde a energia potencial $\pi$ é um polinômio com um ponto de sela na origem, e duas energias cinéticas $T_{1}$ e $T_{2}$, ambas polinômios de segundo grau em $q$ e $\dot{q}$, de forma que a variedade instável do lagrangeano $L_{1}$ tem dimensão 2 , enquanto a variedade instável do lagrangeano $L_{2}$ tem dimensão 1 .

Este exemplo mostra até que ponto a energia cinética pode influir na estabilidade de lagrangeanos analíticos (ou mesmo aqueles em que o jato de ordem $k$ mostra que não tem mínimo) com dois graus de liberdade, pois o teorema central da tese, e o princípio de reversibilidade no tempo das soluções de sistemas lagrangeanos como os considerados, já garantia a existência de uma trajetória assintótica à origem no passado. O exemplo mostra que esta trajetória pode ser única ou não, e isto não depende apenas da energia potencial. É interessante notar que, mesmo sob condições tão restritivas como analiticidade, a caracterização local de um ponto de equilíbrio de um sistema lagrangeano depende também da energia cinética, mesmo que sua estabilidade independa.

Uma possível influência mais forte da energia cinética na estabilidade da origem de lagrangeanos apenas $\mathcal{C}^{k}, \mathcal{C}^{\infty}$ ou mesmo analíticos com mais graus de liberdade não está descartada, e pode 
ser objeto de estudos posteriores. 


\section{Capítulo 2 Trajetórias Assintóticas Resistentes a Pertubações}

\subsection{Breve introdução}

Dado um aberto $\Omega \subset \mathbf{R}^{n}, \mathbf{0} \in \Omega$, e dada uma função $f \in \mathcal{C}\left(\Omega, \mathbf{R}^{n}\right)$, consideramos o campo de vetores

$$
\dot{X}=f(X), f(\mathbf{0})=\mathbf{0} .
$$

onde assumiremos a existência, unicidade e dependência contínua das soluções com respeito aos valores iniciais.

Diremos que uma solução $\phi(t)$ deste campo é uma trajetória assintótica à origem se $\lim _{t \rightarrow+\infty} \phi(t)=0$. No decorrer deste capítulo, estaremos interessados principalmente no caso em que existe uma direção tangente à trajetória, isto é, existe $\lim _{t \rightarrow+\infty} \frac{\phi(t)}{\|\phi(t)\|}$.

Neste capítulo estudaremos trajetórias assintóticas a um ponto de equilíbrio de um campo de vetores, que sejam resistentes a pequenas perturbações do mesmo. Admitiremos que a origem é o ponto de equilíbrio que pretendemos estudar.

O resultado mais famoso nesta linha é o teorema de Hartman. Além deste, também é muito conhecido o fato de que, quando a parte linear na origem de um campo de vetores continuamente 
diferenciável possui um autovalor real e negativo, e se $x$ é um auto-vetor associado a este autovalor, então existe uma trajetória assintótica à origem, tangente a $x$.

Outros trabalhos interessantes foram realizados por Takens e Dumortier( ver [Tak] e [D]). O enfoque principal destes trabalhos, nesta direção, busca, em situações onde $f \in \mathcal{C}^{\infty}$ ou, em alguns casos, $f \in \mathcal{C}^{k}$, condições para que o polinômio de Taylor do campo na origem determine completamente o comportamento do mesmo em uma vizinhança aberta do equilíbrio.

Nosso interesse neste capítulo será o de estudar o campo de vetores e trajetórias assintóticas apenas num "cone" envolvendo uma direção. Nós também não exigiremos quase nenhuma diferenciabilidade das funções que definem o campo de vetores.

Vale lembrar que o estudo de singularidades de campos de vetores com a parte linear degenerada é consideravelmente complicado, e quando este campo não tem boas propriedades de diferenciação, técnicas analíticas como teoremas do ponto fixo ou da função implícita nem sempre funcionam bem.

Nossa saída para este problema foi a utilização de funções auxiliares, uma técnica tradicional desenvolvida nos trabalhos de Liapounof e Četaev, e aprimorada nos trabalhos de Rouche-HabbetsLaloy e Krasovsky (Ver [RHL] e [Kra]).

$\mathrm{Na}$ primeira seção desenvolvemos um novo teorema utilizando funções auxiliares (Teorema 2.3), além de oferecermos novas demonstrações (possivelmente mais simples) de resultados já conhecidos como os teoremas 2.1 e 2.2 .

A segunda seção trabalha com campos de vetores bi-dimensionais e a última seção do capítulo trata de campos tri-dimensionais.

Em todos os campos considerados neste capítulo admitiremos que vale algum teorema de existência, unicidade e dependência 
contínua das soluções com relação às condições iniciais.

Em vários momentos diferentes da tese, especialmente neste Capítulo e no Capítulo 4, utilizaremos implicitamente o seguinte Lema de comparação, de demonstração padrão:

Lema 2.1 Sejam $f \in \mathcal{C}^{1}(\mathbf{R}, \mathbf{R})$ e $g \in \mathcal{C}^{1}(\mathbf{R} \times \mathbf{R}, \mathbf{R})$. Suponha que, para todo $x \in \mathbf{R}$ e todo $t \in \mathbf{R}$, vale que $f(x)>g(x, t)$. Se $x_{1}(t)$ é a solução maximal do problema de Cauchy

$$
\begin{aligned}
\dot{x}(t) & =f(x) \\
x\left(t_{0}\right) & =x_{0}
\end{aligned}
$$

e se $x_{2}(t)$ é a solução maximal de

$$
\begin{aligned}
\dot{x}(t) & =g(x, t) \\
x\left(t_{0}\right) & =x_{0}
\end{aligned}
$$

então, em qualquer instante $t>t_{0}$ onde ambas as soluções estejam definidas, vale que $x_{1}(t)>x_{2}(t)$.

\subsection{Trajetórias Assintóticas e Funções Auxi- liares}

O objetivo desta primeira seção é apresentar teoremas que garantam a existência de trajetórias assintóticas, utilizando para tanto funções auxiliares.

Apresentaremos 3 teoremas, cada um com uma interpretação geométrica diferente. Neles, construiremos um tronco de "cone" com vértice na origem, e estudaremos o que acontece com o campo nas faces "laterais" deste cone. Em todos os teoremas desta seção, $A$ poderá ser entendido como a base deste cone. No primeiro teorema (Teorema 2.1), o campo aponta para dentro do cone em 
toda a sua lateral, representada neste caso por $B$. Nos teoremas posteriores, trataremos dos casos em que o campo sempre aponta para fora do cone na lateral (região outra vez representada por $B$ nas hipóteses do Teorema 2.2), ou de quando existem regiões em que o campo aponta para dentro e regiões em que aponta para fora (regiões representadas por $B$ e $C \cup D$, respectivamente, nas hipóteses do Teorema 2.3).

Teorema 2.1 Sejam $\Omega_{1}, \Omega_{2}$ dois abertos conexos de $\mathbf{R}^{n}, \Omega_{1}$ relativamente compacto e $V_{1}, V_{2} \in \mathcal{C}^{1}\left(\Omega_{2}, \mathbf{R}\right)$. Suponha que $0 \in \partial \Omega_{1}$ $e$

1. $\partial \Omega_{1} \backslash\{0\}=A \cup B, 0 \notin \bar{A}$.

2. $\Omega_{2} \supset \overline{\Omega_{1}} \backslash\{0\}$.

3. $\left.V_{1}\right|_{A}=K_{1}, V_{1}(x)<K_{1}, \forall x \in \Omega_{1}$.

4. $\dot{V}_{1}(x)<0, \forall x \in \Omega_{1}$.

5. $\left.V_{2}\right|_{B}=K_{2}, V_{2}(x)<K_{2}, \forall x \in \Omega_{1}$.

6. $\dot{V}_{2}(x)<0, \forall x \in B$.

Então toda solução $\phi(t)$ de 2.1 tal que $\phi(0) \in \Omega_{1}$ é assintótica à origem.

Prova: Da limitação de $\Omega_{1}$ e das hipóteses 3 e 4 , segue que toda solução $\phi(t)$ começando em $\Omega_{1}$, ou sai deste aberto, ou tem um ponto de acumulação no seu bordo. As mesmas hipóteses claramente impedem que uma solução saia por $A$ ou tenha em $A$ algum ponto de acumulação. Da mesma forma, as hipóteses 5 e 6 não permitem que uma solução saia por $B$ ou tenha neste algum ponto de acumulação. 
Logo, como $\partial \Omega_{1}=\{0\} \cup A \cup B$, a única possibilidade restante é que 0 seja o único ponto de acumulação de $\phi(t)$, o que demonstra o resultado.

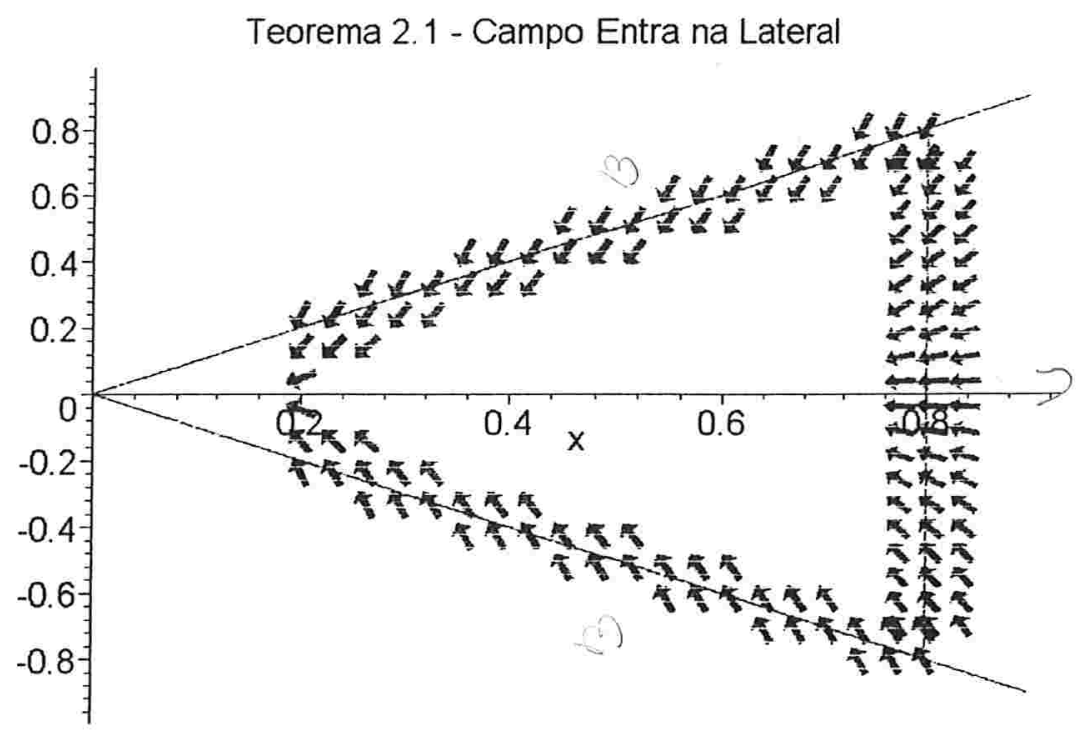

Teorema 2.2 Sejam $\Omega_{1}, \Omega_{2}$ dois abertos conexos de $\mathbf{R}^{n}, \Omega_{1}$ relativamente compacto e $V_{1}, V_{2} \in \mathcal{C}^{1}\left(\Omega_{2}, R\right)$. Suponha que $0 \in \partial \Omega_{1}$ $e$

1. $\partial \Omega_{1} \backslash\{\mathbf{0}\}=A \cup B, \mathbf{0} \notin \bar{A}$.

2. $\Omega_{2} \supset \overline{\Omega_{1}} \backslash\{0\}$.

3. $\left.V_{1}\right|_{A}=K_{1}, V_{1}(x)<K_{1}, \forall x \in \Omega_{1}$.

4. $\dot{V}_{1}(x)<0, \forall x \in \Omega_{1}$.

5. $\left.V_{2}\right|_{B}=K_{2}, V_{2}(x)<K_{2}, \forall x \in \Omega_{1}$.

6. $\dot{V}_{2}(x)>0, \forall x \in B$. 
Então existe uma solução $\phi(t)$ de (2.1) tal que $\phi(0) \in \Omega_{1} e$ $\lim _{t \rightarrow \infty} \phi(t)=0$.

Prova: Tomamos uma sequência de pontos $\left(x_{k}\right)$ tal que $\lim _{k \rightarrow \infty} x_{k}=$ $0, x_{k} \in \Omega_{1}$, para todo $k \in \mathrm{N}$. Sejam então $\phi_{k}(t)$ as soluções da equação 2.1 tais que $\phi_{k}(0)=x_{k}$. Veja que as hipóteses 3 e 4 garantem que existe $t_{k}<0$ tal que $\phi_{k}\left(t_{k}\right) \in \partial \Omega_{1}, \phi_{k}(t) \in \Omega_{1}$, para todo $t \in\left(t_{k}, 0\right]$.

As hipóteses 5 e 6 asseguram que $\phi_{k}\left(t_{k}\right) \in A$, pois o campo aponta para fora em $B$ e $\operatorname{logo}$ uma trajetória que passa por um ponto de $B$ não pode estar em $\Omega_{1}$ num instante posterior suficientemente próximo.

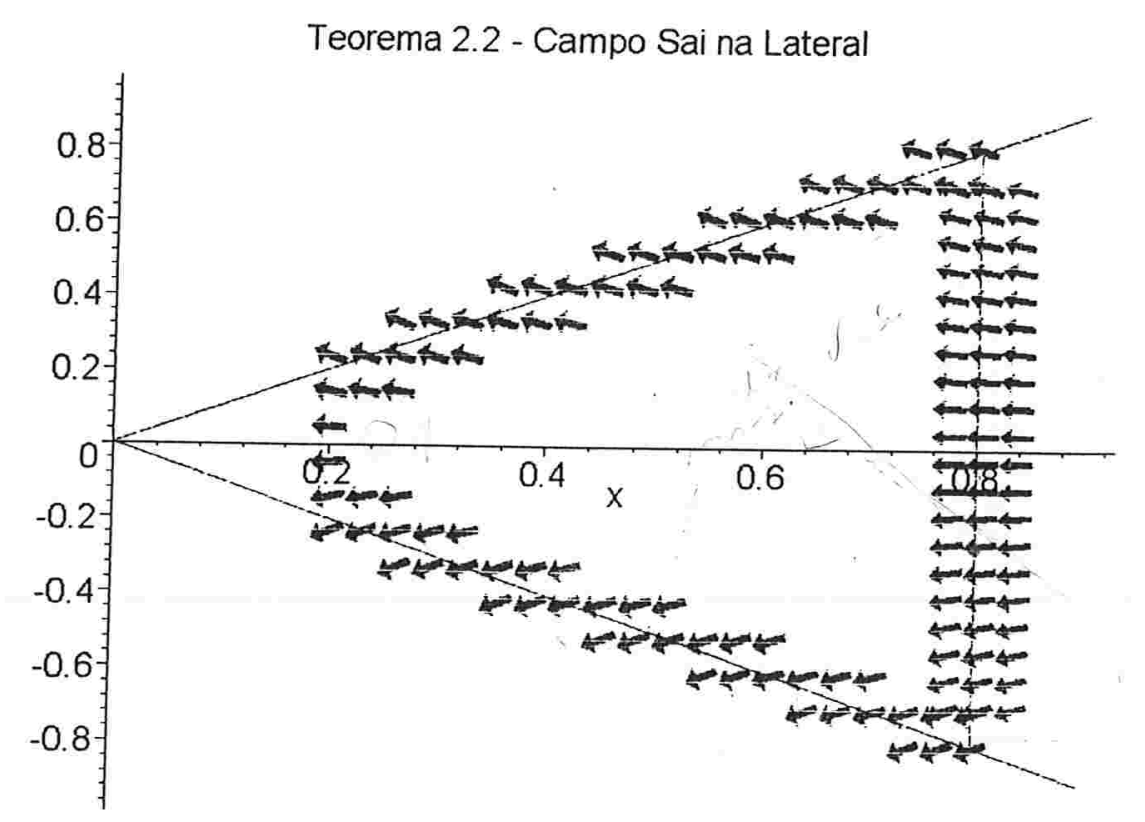

A limitação de $\Omega_{1}$ garante a existência de um ponto $y$ e de uma subsequência $n_{k}$ tal que $\lim _{k \rightarrow \infty} \phi_{n_{k}}\left(t_{n_{k}}\right)=y$. Claramente $y \in \bar{A}$ e portanto $y \neq 0$. 
Seja $\phi$ a solução que satisfaz $\phi(0)=y$. Afirmamos que $\phi$ é assintótica à origem. Note que, se por absurdo isto fosse falso, as hipóteses 3 e 4 garantiriam a existência de um instante $\bar{t}>0$ com a propriedade $\phi(\bar{t}) \notin \overline{\Omega_{1}}$.

Como $\phi$ é contínua, a função $\|\phi(t)\|$ tem um ponto de mínimo local em $[0, \bar{t}]$. Seja $\varepsilon$ o valor da função neste ponto.

Visto que $\phi_{n_{k}}\left(t_{n_{k}}\right)$ converge para $y$, a dependência contínua das soluções nos garante que as funções $\phi_{n_{k}}\left(t^{\prime}+{ }^{\prime} t_{n_{k}}\right)$ convergem uniformemente, no intervalo $[0, \bar{t}]$, para a função $\phi$. Portanto, existe $k_{0} \in \mathrm{N}$ tal que, para $k>k_{0},\left\|\phi_{n_{k}}(t)\right\|>\frac{\varepsilon}{2}$, para todo $t \in\left[t_{n_{k}}, t_{n_{k}}+\bar{t}\right]$.

Visto que $\lim _{k \rightarrow \infty} \phi_{k}(0)=\lim _{k \rightarrow \infty} x_{k}=0$, temos que para $k$ suficientemente grande $0>t_{n_{k}}+\bar{t}$. Por outro lado, como $\phi(\bar{t}) \notin \overline{\Omega_{1}}$, para $k$ suficientemente grande $\phi_{n_{k}}\left(t_{n_{k}}+\bar{t}\right) \notin \overline{\Omega_{1}}$, absurdo, dado que $t_{n_{k}}+\bar{t} \in\left[t_{n_{k}}, 0\right]$.

Teorema 2.3 Sejam $\Omega_{1}, \Omega_{2}$ dois abertos conexos de $\mathbf{R}^{n}, \Omega_{1}$ relativamente compacto e $V_{1}, V_{2}, V_{3} \in \mathcal{C}^{1}\left(\Omega_{2}, R\right)$. Suponha que $\mathbf{0} \in$ $\partial \Omega_{1} e$

1. $\partial \Omega_{1} \backslash\{0\}=A \cup B \cup C \cup D, 0 \notin \bar{A}$.

2. $\Omega_{2} \supset \overline{\Omega_{1}} \backslash\{0\}$.

3. $\left.V_{1}\right|_{A}=K_{1}, V_{1}(x)<K_{1}, \forall x \in \Omega_{1}$.

4. $\dot{V}_{1}(x)<0, \forall x \in \Omega_{1}$.

5. $\left.V_{2}\right|_{B}=K_{2}, V_{2}(x)<K_{2}, \forall x \in \Omega_{1}$.

6. $\dot{V}_{2}(x)<0, \forall x \in B$.

7. $\bar{C} \cap \bar{D}=\{0\},\left.e V_{3}\right|_{C}=\left.V_{3}\right|_{D}=K_{3}, V_{3}(x)<K_{3}, \forall x \in \Omega_{1}$. 
8. $\dot{V}_{3}(x)>0, \forall x \in C \cup D$.

Então existe uma solução $\phi(t)$ de (2.1) tal que $\phi(0) \in \Omega_{1} \epsilon$ $\lim _{t \rightarrow \infty} \phi(t)=0$.

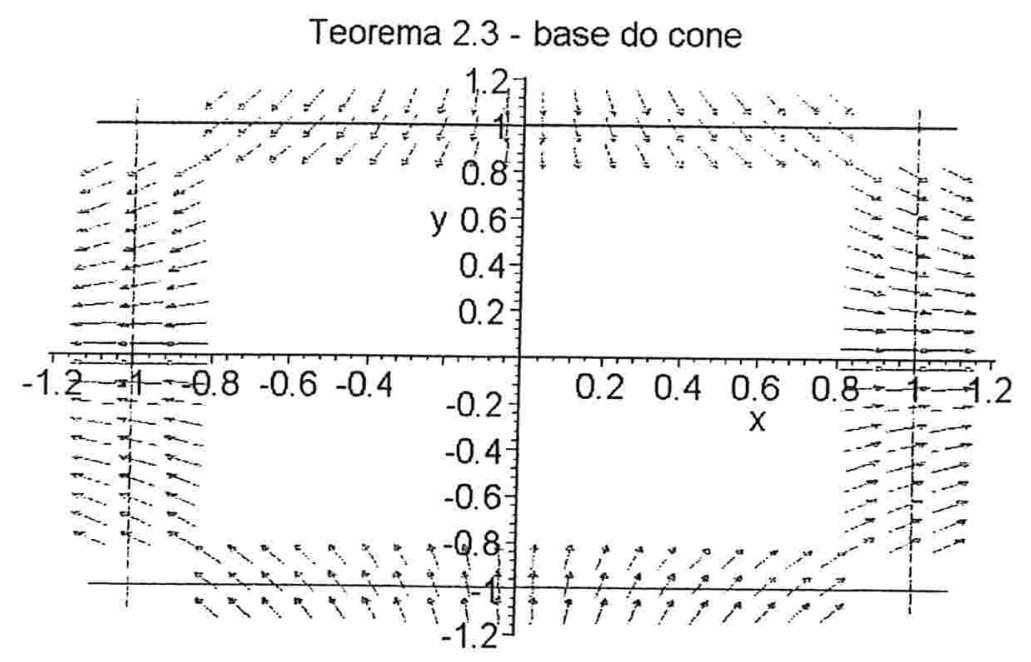

Prova: Começamos definindo a função $H: \Omega_{1} \rightarrow \overline{\mathbf{R}}$ por

$$
H(x)=\sup \left\{\tau>0: \phi_{x}(t) \in \Omega_{1} ; t \in[0, \tau]\right\}
$$

ou $H(x)=+\infty$ caso o supremo não exista, onde $\phi_{x}(t)$ é a solução de (2.1) que satisfaz $\phi(0)=x$.

Note que as hipóteses 3 e 4 garantem que, se existe $z$ tal que $H(z)=+\infty$, então o $\omega$-limite de $\phi_{z}(t) \subset \partial \Omega_{1}$. As hipóteses 3 a 8 mostram que o único conjunto positivamente invariante contido em $\partial \Omega_{1}$ é $\{0\}$, logo temos que $\lim _{t \rightarrow \infty} \phi_{z}(t)=\mathbf{0}$.

As hipóteses 3 a 6 impedem que $\phi_{x}(H(x))$ pertença a $A \cup B$. 
Como $\dot{V}_{3} \neq 0$ em $C \cup D$, e $V_{3}$ é constante em $C \cup D$, o campo de vetores é necessariamente transversal a $C \cup D$ (Note que $V_{3}$ é continuamente diferenciável e $\nabla V_{3} \neq 0$ em $C \cup D$, portanto podemos falar em transversalidade do campo de vetores com $C \cup$ $D)$.

Esta condição de transversalidade mostra que se $H(x)=a$, então $\phi_{x}(a) \in C \cup D$, portanto existe $\varepsilon>0$ tal que $\phi_{x}(t) \notin \bar{\Omega}_{1}$, para todo $t \in(a, a+\varepsilon]$. Este fato, e a dependência contínua das soluções com relação às condições iniciais, garantem que $H$ é finita num aberto, e é contínua no mesmo.

Seja agora $\gamma:[0,1] \rightarrow \overline{\Omega_{1}}$, contínua,

$$
\gamma(0) \in C, \gamma(1) \in D, \gamma(s) \in \Omega_{1}, \forall s \in(0,1) .
$$

Tal curva existe, visto que $\Omega_{1}$ é aberto e conexo. Afirmamos que existe $c \in(0,1)$ tal que $\phi_{\gamma(c)}(t)$ é assintótica à origem.

Suponha por absurdo que isto seja falso. Então a função

$$
\begin{aligned}
G & :[0,1] \rightarrow \partial \Omega_{1} \\
G(s) & =\phi_{\gamma(s)} \circ H(\gamma(s))
\end{aligned}
$$

estaria bem definida e seria contínua.

Vimos que as hipóteses 3 a 6 mostram que $G(s) \in C \cup D$. Por último, temos que $G(0) \in C$ e $G(1) \in D$. A hipótese 7 então nos mostraria que a imagem por $G$ do intervalo $[0,1]$ não seria conexa, o que é claramente um absurdo.

\subsection{Estudo de trajetórias assintóticas em duas dimensões}

Nosso objetivo nesta seção será estudar sob que hipóteses uma trajetória $\phi(t)$, assintótica à origem e tangente a uma direção 
fixa, solução de um campo de vetores $V(x, y) \in \mathcal{C}^{k}, k \geq k_{0} \geq 2$, definido num aberto de $\mathbf{R}^{2}$, de alguma forma persiste quando este mesmo campo sofre uma "pequena perturbação".

De maneira um pouco mais formal, queremos encontrar condições sobre o campo

$$
\begin{array}{r}
\dot{X}=\bar{V}=V+R \\
R(x, y) \in \mathcal{C}^{k}, k \geq k_{0} \geq 2
\end{array}
$$

onde $R$ é pequeno em algum sentido, para que exista uma solução $\bar{\phi}(t)$, assintótica à origem, e que seja tangente à mesma direção que $\phi(t)$.

Para melhor motivarmos o contexto exato em que iremos trabalhar, admitiremos que a nossa solução $\phi(t)=(\bar{x}(t), \bar{y}(t))$ é tal que podemos parametrizar $\bar{y}(t)$ por $\gamma(\bar{x}(t))$, onde a curva $y=\gamma(x)$ é algébrica e $\gamma \in O\left(x^{2}\right)$. Encontraremos uma situação muito parecida com esta na seção 3 do próximo capítulo.

Como $\gamma(x)$ pode ser escrita como uma série de expoentes fracionários, após realizarmos a mudança de coordenadas

$$
x=\bar{x} \quad y=\bar{y}-\gamma(\bar{x})
$$

chegaríamos a um sistema de equações que pode ser expresso como

$$
\begin{aligned}
& \dot{x}=-\alpha_{0} x^{k_{0}}+\alpha_{1} y x^{k_{1}}+R(x, y) \\
& \dot{y}=\beta_{1} y x^{l_{1}}+S(x, y)
\end{aligned}
$$

onde $\alpha_{0}>0, k_{0}, k_{1}, l_{1}$ são reais positivos não nulos com $k_{0} \geq 2$, $\alpha_{1}, \beta_{1}$, são reais, $R \in O\left(x^{k_{0}}\right)+o\left(y x^{k_{1}}\right)+O\left(y^{2}\right)$, e $S \in O\left(x^{c}\right)+$ $o\left(y x^{l_{1}}\right)+O\left(y^{2}\right)$. 
Este será o sistema que estudaremos. Conceitualmente, como a trajetória $\phi$ original era tangente ao semi-eixo $x \geq 0$, também será nesta direção que procuraremos a nossa trajetória $\bar{\phi}$. Vale notar que, da maneira em que expusemos o sistema (2.3), as principais dependências do campo na direção $x$ (direção tangente à trajetória assintótica) ficaram explícitas, porém não possuimos quase nenhuma informação nas outras direções.

Por outro lado, como estamos interessados em trajetórias tangentes à semireta $\{(x, y) \mid y=0, x \geq 0\}$, podemos nos restringir ao semi-plano onde $x \geq 0$, o que elimina ambiguidades relacionadas ao sinal de termos do tipo $x^{r} \operatorname{com} r$ real.

Por simplicidade, admitiremos ainda que $\alpha_{1}$ e $\beta_{1}$ não são nulos. Os resultados que obteremos nesta seção também são válidos, com pequenas adaptações nas hipóteses e nas demonstrações, nos casos em que $\dot{x}=-\alpha_{0} x^{k_{0}}+o\left(x^{k_{0}}\right)+O\left(y^{2}\right)$ bem como nos casos em que $\dot{y}=O\left(x^{c}\right)+o\left(y x^{k_{0}}\right)+O\left(y^{2}\right)$. Porém, dada a similaridade das provas e com o intuito de não alongarmos desnecessariamente as demonstrações, estes casos não serão considerados.

Lembramos que, quando $R$ e $S$ são nulos, existe uma trajetória assintótica onde $y(t)=0$. Nosso principal objetivo será, com informações sobre $k_{0}, k_{1}, \alpha_{1}, \beta_{1}$ e $l_{1}$, estimar os valores de $c$ que garantam a existência de uma trajetória assintótica tangente ao semi-eixo dos $x$ positivo. Suporemos que $\alpha_{0}=1$, o que claramente poderia ser obtido com uma reparametrização linear do tempo.

Resta fazer um último comentário. Em várias das demonstrações desta seção, trataremos apenas do caso em que $\alpha_{1}>0$. Isso poderia ser obtido com a mudança de coordenadas $z=-y$. Através desta simples mudança, o leitor poderá verificar que não há restrição de generalidade nos teoremas seguintes em que apenas estes casos são considerados. 
O principal teorema desta seção é

Teorema 2.4 No sistema (2.3), se

$$
c>\tilde{c}=\max \left(\begin{array}{r}
\min \left(2 k_{0}-1-k_{1}, k_{0}+l_{1}-k_{1}\right), \\
\min \left(\frac{3 k_{0}}{2}-1, \frac{k_{0}}{2}+l_{1}\right), \\
\min \left(2 k_{0}-2,2 l_{1}\right)
\end{array}\right)
$$

então, para quaisquer $R, S$ nas condições descritas acima, existe uma solução assintótica à origem e tangente ao semi-eixo $x \geq 0$, com ordem de contato maior que

$$
d=\max \left(k_{0}-k_{1}, \frac{k_{0}}{2}, \min \left(k_{0}-1, l_{1}\right)\right) \geq 1 .
$$

Prova: Escolhemos primeiro $\tilde{d} \in(d, d+c-\tilde{c})$ tal que $\tilde{d} \neq \beta_{1}$. Definimos em $\Omega_{2}=\{(x, y) \mid x>0\}$,

$$
\begin{aligned}
V_{1}(x, y) & =x \text { e } V_{2}(x, y)=\left(\frac{y}{x^{\tilde{d}}}\right)^{2}, \\
\Omega_{1} & =\left\{(x, y) \mid 0<x<\delta, V_{2}(x, y)<1\right\}, \\
A & =\left\{(x, y) \mid x=\delta \text { e } V_{2}(x, y) \leq 1\right\}, \\
B & =\left\{(x, y) \mid 0<x<\delta \text { e } V_{2}(x, y)=1\right\} .
\end{aligned}
$$

e o número $\delta>0$ será suficientemente pequeno, escolhido de forma conveniente posteriormente.

Mostraremos que:

1. Se $l_{1}>k_{0}-1$, ou se $\beta_{1}>0$, ou, ainda, se $l_{1}=k_{0}-1 \mathrm{e}$ $-\beta_{1}<\tilde{d}$, então a função $V_{2}$ que definiremos em (2.5) e o campo (2.3) satisfazem as hipóteses do Teorema 2.2.

2. Se $l_{1}<k_{0}-1$ e $\beta_{1}<0$, ou se $l_{1}=k_{0}-1$ e $-\beta_{1}>\tilde{d}$, então a função $V_{2}$ que definiremos em (2.5) e o campo (2.3) satisfazem as hipóteses do Teorema 2.1. 
Note que isto cobre todos os casos, pois escolhemos $\tilde{d} \neq-\beta_{1}$. Portanto, como ambos os teoremas garantem a existência de uma trajetória assintótica à origem que permanece em $\Omega_{1}$, uma parte das conclusões já estará assegurada.

Finalmente, obteremos facilmente a estimativa sobre a ordem de contato enunciada no teorema através da construção de $\Omega_{1}$, claramente tangente ao semi-eixo com ordem maior que $d$.

Portanto, para terminarmos a demonstração, só necessitamos provar as duas afirmações anteriores.

Nossa principal tarefa será estimar $\dot{V}_{1}(x, y)=\dot{x}$ em $\Omega_{1}$, e $\dot{V}_{2}(x, y)$ quando $(x, y) \in B$. Mas veja que

$$
\begin{aligned}
\dot{V}_{2}(x, y)= & 2\left(\frac{y}{x^{\tilde{d}}}\right) \frac{\dot{y} x^{\tilde{d}}-\tilde{d} x^{\tilde{d}-1} \dot{x} y}{x^{2 \tilde{d}}}= \\
= & \frac{2}{x^{3 \tilde{d}}}\left[y x^{\tilde{d}} S(x, y)+y^{2}\left(\beta_{1} x^{\tilde{d}+l_{1}}+\tilde{d} x^{\tilde{d}+k_{0}-1}-\right.\right. \\
& \left.\quad-\tilde{d} R(x, y))-y^{3} x^{k_{1}+\tilde{d}-1}\right]
\end{aligned}
$$

e, como $x>0$, o sinal de $\dot{V}_{2}$ será determinado por $y x^{\tilde{d}} S(x, y)+y^{2}\left(\beta_{1} x^{\tilde{d}+l_{1}}+\tilde{d} x^{\tilde{d}+k_{0}-1}-\tilde{d} x^{\tilde{d}-1} R(x, y)\right)-y^{3} x^{k_{1}+\tilde{d}-1}$.

Quando substituírmos $y=a x^{\tilde{d}}, a= \pm 1$, na fórmula acima, obteremos

$$
\begin{array}{r}
a x^{2 \tilde{d}} S\left(x, a x^{\tilde{d}}\right)+a^{2}\left(\beta_{1} x^{3 \tilde{d}+l_{1}}+\tilde{d} x^{3 \tilde{d}+k_{0}-1}-\tilde{d} x^{3 \tilde{d}-1} R\left(x, x^{\tilde{d}}\right)\right)- \\
-a^{3} x^{k_{1}+4 \tilde{d}-1} .
\end{array}
$$

Se lembrarmos que em $\Omega_{1}$ vale que $y^{2}<x^{2 \tilde{d}}$, teremos de (2.6) que o sinal de $\dot{V}_{2}$ é dado por:

$$
\begin{aligned}
a^{2}\left(\beta_{1} x^{3 \tilde{d}+l_{1}}-\tilde{d} x^{3 \tilde{d}+k_{0}-1}\right)+O\left(x^{2 \tilde{d}+c}\right)+ & O\left(x^{4 \tilde{d}}\right)+O\left(x^{4 \tilde{d}-1+k_{1}}\right)+ \\
& +o\left(x^{3 \tilde{d}+l_{1}}\right)+o\left(x^{3 \tilde{d}+k_{0}-1}\right)
\end{aligned}
$$


e, como $\tilde{d}>\min \left(l_{1}, k_{0}-1\right)$, temos que $O\left(x^{4 \tilde{d}}\right)=o\left(\beta_{1} x^{3 \tilde{d}+l_{1}}\right)+$ $o\left(\tilde{d} x^{3 \tilde{d}+k_{0}-1}\right)$. Além disso, como $\tilde{d}>k_{0}-k_{1}$, temos também que $O\left(x^{4 \tilde{d}-1+k_{1}}\right)=o\left(x^{3 \tilde{d}+k_{0}-1}\right)$. Finalmente, de $\tilde{d}-d<c-\tilde{c}$, temos que $c-\tilde{d}>\tilde{c}-d=\min \left(k_{0}-1, l_{1}\right)$, e disto segue finalmente que

$$
O\left(x^{2 \tilde{d}+c}\right)=o\left(\beta_{1} x^{3 \tilde{d}+l_{1}}+\tilde{d} x^{3 \tilde{d}+k_{0}-1}\right) .
$$

Portanto fica claro que, tomando $\delta$ suficientemente pequeno, o sinal de $\dot{V}_{2}$ quando restrito a $B$, é completamente determinado pelo sinal de $\beta_{1} x^{3 \tilde{d}+l_{1}}+\tilde{d} x^{3 \tilde{d}+k_{0}-1}$.

Como conseqüência, se $l_{1}>k_{0}-1$, ou se $\beta_{1}>0$, ou se $l_{1}=$ $k_{0}-1$ e $-\beta_{1}<\tilde{d}$, então $\dot{V}_{2}(x)>0, \forall x \in B$.

Se, ao contrário, $l_{1}<k_{0}-1$ e $\beta_{1}<0$, ou se $l_{1}=k_{0}-1$ e $-\beta_{1}>\tilde{d}$, então $\dot{V}_{2}(x)<0, \forall x \in B$.

Por último, falta analisar o sinal de $\dot{x}$ em $\Omega_{1}$, mas note que

$$
\dot{x}=-x^{k_{0}}+O\left(y x^{k_{1}}\right)+O\left(y^{2}\right)+o\left(x^{k_{0}}\right),
$$

que em $\Omega_{1}$ pode ser escrito como

$$
\dot{x}=-x^{k_{0}}+O\left(x^{k_{1}+\tilde{d}}\right)+O\left(x^{2 \tilde{d}}\right)+o\left(x^{k_{0}}\right)=-x^{k_{0}}+o\left(x^{k_{0}}\right) .
$$

Esta última igualdade mostra que $\dot{V}_{1}<0$, para todo $x$ em $\Omega_{1}$, e demonstra o resultado.

Corolário 2.1 No sistema (2.3), se $c>2 k_{0}-1$, então existe solução de (2.3) assintótica à origem e tangente ao semi-eixo $x \geq$ 0 .

Prova: Basta verificar que $2 k_{0}-1 \geq \tilde{c}$ no último teorema.

Iremos mostrar que as estimativas sobre $c$ enunciadas no Teorema 2.4 são as melhores possíveis em boa parte dos casos cobertos pelo mesmo, porém ainda cabem mais dois teoremas que melhorarão estas estimativas em alguns casos particulares, sob hipóteses um pouco mais restritivas de regularidade dos restos $R$ e $S$. 
Teorema 2.5 No sistema (2.3) suponha que $\beta_{1}<0, l_{1}<k_{0}-1$, $k_{1}<\frac{k_{0}}{2}$, e que $S(x, y)=f(x)+o\left(y x^{l_{1}}\right)+O\left(y^{2}\right)$, onde $f(x)=$ $a x^{c}+o\left(x^{c}\right), a \neq 0$. Suponha ainda que $\alpha_{1} a<0$. Se

$$
c>\bar{c}=\max \left(l_{1}, l_{1}+1-k_{1}, 1, k_{1}\right)+l_{1},
$$

então existe uma solução de (2.3) assintótica à origem, tangente à semi-reta $x \geq 0$.

Prova: A demonstração será feita, sem perda de generalidade, para o caso em que $a>0$, e as idéias envolvidas serão as mesmas do teorema passado.

Seja $d<c-l_{1}, d>\bar{c}-l_{1} \geq 1$, e considere as funções

$$
V_{1}(x, y)=x, V_{2}(x, y)=y^{2}-y x^{d},
$$

definidas em $\Omega_{2}=\{(x, y) \mid x>0\}$. Seja também

$$
\Omega_{1}=\left\{(x, y) \mid 0<x<\delta, V_{2}(x, y)<0\right\},
$$

e note que

$$
\dot{V}_{2}=\left(2 y \dot{y}-\dot{y} x^{d}-d y x^{d-1} \dot{x}\right) .
$$

Quando $V_{2}=0$, temos que ou $y=0$, ou então $y=x^{d}$.

Se calcularmos

$$
\dot{V}_{2}(x, 0)=-\dot{y} x^{d},
$$

então para $\delta>0$ suficientemente pequeno e $0<x<\delta, \dot{V}_{2}(x, 0)$ é negativa.

Por outro lado, se calcularmos

$$
\begin{aligned}
\frac{\dot{V}_{2}\left(x, x^{d}\right)}{x^{d}}= & -\dot{y}-d x^{d-1} \dot{x}= \\
= & a x^{c}+\beta_{1} x^{d+l_{1}}+d x^{k_{0}+d-1}-d \alpha_{1} x^{k_{1}+2 d-1}+ \\
& +O\left(x^{2 d}\right)+\text { tos }
\end{aligned}
$$


onde tos significa termos de ordem superior a

$$
\max \left(c, d+l_{1}, d+k_{0}-1, k_{1}+2 d-1\right) .
$$

Se notarmos que $d+l_{1}<c$, que $d>l_{1}$ e que

$$
2 d+k_{1}-1=d+d+k_{1}-1>d+l_{1},
$$

temos que $\dot{V}_{2}\left(x, x^{d}\right)=\beta_{1} x^{l_{1}+2 d}+o\left(x^{l_{1}+2 d}\right)$.

Como $\beta_{1}<0$ segue que, eventualmente diminuindo $\delta, \dot{V}_{2}\left(x, x^{d}\right)$ é negativa, para todo $x \in(0, \delta)$.

Além disso, se $(x, y) \in \Omega_{1}$, vale que

$$
\begin{aligned}
\dot{V}_{1}(x, y) & =\dot{x}=-x^{k_{0}}+\alpha_{1} y x^{k_{1}}+O\left(y^{2}\right)+\text { tos }= \\
& =-x^{k_{0}}+\alpha_{1} y x^{k_{1}}+O\left(x^{2 d}\right)+\text { tos },
\end{aligned}
$$

e como $d>k_{1}, y>0$ e $\alpha_{1}<0$, temos para $\delta$ suficientemente pequeno que $y^{2}$ tem ordem menor do que $y x^{k_{1}}$ e portanto,

$$
\dot{V}_{1}(x, y)<0, \forall(x, y) \in \Omega_{1} .
$$

Logo, aplicando diretamente o Teorema 2.1, demonstramos o resultado.

Teorema 2.6 No sistema (2.3) suponha que $\beta_{1}<0, k_{1}<1, k_{1}<$ $l_{1}<k_{0}-1$, e que $S(x, y)=f(x)+o\left(y x^{l_{1}}\right)+O\left(y^{2}\right)$, onde $f(x)=$ $a x^{c}+o\left(x^{c}\right), a \neq 0$. Suponha ainda que $\alpha_{1} a>0$ e $c>k_{1}+1$. Então existe uma solução assintótica à origem, tangente à semireta $x \geq 0$.

Esboço de Prova: Como as idéias aqui presentes são as mesmas dos dois últimos teoremas, iremos apenas esboçar uma demonstração. Sem perda de generalidade, trataremos do caso em que $a>0$. 
Primeiro tomamos $d \in\left(1, \frac{c-k_{1}+1}{2}\right)$.

Consideramos as funções $V_{1}, V_{2}$ definidas na faixa

$$
\Omega_{2}=\{(x, y) \mid 0<x<\delta\}
$$

por

$$
\begin{aligned}
& V_{1}(x, y)=x, \\
& V_{2}(x, y)=y^{2}+y x^{d},
\end{aligned}
$$

e definimos $\Omega_{1}=\left\{(x, y) \mid x>0, V_{2}(x, y)<0\right\}$.

Derivando pelas trajetórias a função $V_{2}(x, y)$, quando restrita à $y=0$, obtemos

$$
\dot{V}_{2}(x, 0)=\dot{y} x^{d},
$$

que é positiva se $\delta$ for suficientemente pequeno.

Também derivando no tempo a função $V_{2}$ quando restrita à curva $y=-x^{d}$, obtemos

$$
\begin{aligned}
\frac{\dot{V}_{2}\left(x,-x^{d}\right)}{x^{d}}= & d x^{d-1} \dot{x}-\dot{y}= \\
= & d x^{k_{0}+d-1}+d \alpha_{1} x^{k_{1}+2 d-1}-a x^{c}+\beta_{1} x^{d+l_{1}}+ \\
& O\left(x^{2 d}\right)+\text { tos. }
\end{aligned}
$$

Uma análise simples mostra que o termo dominante na última expressão é $d \alpha_{1} x^{k_{1}+2 d-1}$, que para $\delta$ pequeno também é positivo.

Também verifica-se facilmente que, restrito a $\Omega_{1}, \dot{x}$ é negativo, e assim podemos aplicar o Teorema 2.2, demonstrando o resultado.

Os próximos teoremas mostrarão que estes resultados não podem ser muito melhorados, e que as estimativas para $c$ são ótimas para os casos cobertos pelos três teoremas acima. 
Teorema 2.7 Se $c=\min \left(2 k_{0}-2,2 l_{1}\right)$ então, para qualquer sistema do tipo (2.3) existe uma função $S(x, y)=O\left(x^{c}\right)+O\left(y^{2}\right)$, tal que nenhuma solução é assintótica à origem e tangente ao semi-eixo $x \geq 0$.

Prova: Trataremos do caso particular em que $\alpha_{1}>0, \beta_{1}>0$. O leitor não terá dificuldade em adaptar o exemplo para os casos restantes.

Considere o sistema

$$
\begin{aligned}
& \dot{x}=-x^{k_{0}}+\alpha_{1} y x^{k_{1}} \\
& \dot{y}=-a y^{2}-a x^{c}-\frac{\beta_{1}}{2} y^{2}+\beta_{1} y x^{l_{1}}-\frac{\beta_{1}}{2} x^{2 l_{1}}
\end{aligned}
$$

onde $a>k_{0}-1$ é uma constante e veja que $S(x, y)$ está definida pela segunda equação.

Claramente $\dot{y}<0$, logo, se uma solução $\phi(t)=(x(t), y(t))$ satisfaz $y(s)<0$ para algum instante $s$, então esta solução não é assintótica à origem.

Suponha, por absurdo, que existe uma solução de (2.12) assintótica à origem, tal que $x(t)>0$, para todo $t \in[0, \infty)$. Para tal solução, $y(t)>0$, para todo $t \in[0, \infty)$, e $\log \dot{x}>$ $-x^{k_{0}}$. Como a solução de $\dot{z}=-z^{k_{0}}$, com $z(0)=z_{0}>0$, é $z(t)=\left(z_{0}^{1-k_{0}}+\left(k_{0}-1\right) t\right)^{\frac{-1}{k_{0}-1}}$ temos que, se $x(0)=x_{0}$,

$$
x(t)>\left(x_{0}^{1-k_{0}}+\left(k_{0}-1\right) t\right)^{\frac{-1}{k_{0}-1}}, \forall t>0 .
$$

Por outro lado $\dot{y}<-a y^{2}$, e portanto, se $y(0)=y_{0}$, segue pelo raciocínio anterior que

$$
y(t)<\left(y_{0}^{-1}+a t\right)^{-1} .
$$


Vale notar que como $y(t)$ é decrescente e limitada por 0 , o $\lim _{t \rightarrow \infty} y(t)$ tem que existir.

Finalmente, lembramos que $\dot{y}<-a x^{c} \leq-a x^{2\left(k_{o}-1\right)}$, logo

$$
\begin{aligned}
\lim _{t \rightarrow \infty} y(t)>y\left(t_{0}\right) & +\int_{t_{0}}^{\infty}-a x(s)^{2\left(k_{0}-1\right)} d s>y\left(t_{0}\right)+ \\
& +\int_{t_{0}}^{\infty}-a\left(x_{0}^{1-k_{0}}+\left(k_{0}-1\right) s\right)^{-2} d s, \forall t_{0}>0 .
\end{aligned}
$$

Esta última equação e o fato de que $y(t)>0$ implicam em

$$
\left(y_{0}^{-1}+a t\right)^{-1}-\frac{a}{k_{0}-1}\left(x_{0}^{1-k_{0}}+\left(k_{0}-1\right) t\right)^{-1}>0, \forall t>0,
$$

o que só pode ocorrer se $a \leq k_{0}-1$, o que configura um absurdo visto que $a>k_{0}-1$.

Outro resultado na mesma direção é

Teorema $2.8 S e k_{1}>\frac{k_{0}}{2}$ e $c=\min \left(3 \frac{k_{0}}{2}-1, \frac{k_{0}}{2}+l_{1}\right)$, então existem $S(x, y), R(x, y)$, tais que nenhuma solução de (2.3) assintótica à origem é tangente ao semi-eixo $\{(x, y) \mid y=0, x \geq 0\}$.

Prova: Seremos um pouco menos formais aqui do que no resultado anterior, pois as idéias envolvidas são exatamente as mesmas. Suponha, sem perda de generalidade, que $\alpha_{1}>0$. Considere o seguinte sistema:

$$
\begin{aligned}
& \dot{x}=-x^{k_{0}}+\alpha_{1} y x^{k_{1}}+\left(1+\alpha_{1}\right) y^{2} \\
& \dot{y}=-y^{2}+\beta_{1} y x^{l_{1}}-a x^{c}
\end{aligned}
$$

onde $a>\left|\beta_{1}\right|+k_{0} / 2$ é uma constante positiva, e suponha por absurdo que existe uma solução $\phi(t)=(x(t), y(t))$ assintótica à origem e tangente ao semi eixo $x>0$. 
Note que se $|y|>x^{\frac{k_{0}}{2}}$ então $\dot{x}>0$. Além disso, se $|y|<\left|\frac{a}{\beta_{1}}\right| x^{\frac{k_{0}}{2}}$, temos que $\dot{y}<0$. Destas observações segue que, para todo $t>0$, temos que $y(t)>0$ pois, se $y\left(t_{0}\right) \leq 0$, então para todo $t \in\left[t_{0}, \infty\right)$, temos que ou $y(t)$ está decrescendo se $y>-x^{\frac{k_{0}}{2}}$, ou $x(t)$ está crescendo se $y<-x^{\frac{k_{0}}{2}}$.

Logo, a solução precisaria cortar a curva $y=-x^{\frac{k_{0}}{2}}$ infinitas vezes para se aproximar da origem. Mas, com as propriedades de crescimento descritas, intersecções consecutivas da solução com a curva estão progressivamente mais distantes da origem, o que impede que a trajetória seja assintótica à mesma.

Para completarmos a demonstração, o raciocínio será muito similar ao visto no último teorema.

Primeiro, notamos que existe $t_{1}$ tal que $y\left(t_{1}\right)<x\left(t_{1}\right)^{\frac{k_{0}}{2}}$, caso contrário $x$ seria estritamente crescente. Notemos também que para $t \in\left[t_{1}, \infty\right), y(t)<x(t)^{\frac{k_{0}}{2}}$, visto que o campo de vetores cruza esta curva transversalmente em todos os pontos para os quais $x>0$, portanto uma solução não pode interceptar a curva em 2 pontos distintos.

Vale notar que, como $y(t)>0$, temos que $\dot{x}>-x^{k_{0}}$. Por outro lado, para $t>t_{0}$, temos que

$$
\begin{aligned}
\dot{y}<\left(\beta_{1}-a\right) x^{c} & \leq\left(\beta_{1}-a\right) x^{3 \frac{k_{0}}{2}-1}< \\
<-\left(\beta_{1}-a\right) x^{\frac{k_{0}}{2}-1} \dot{x} & =-\frac{2\left(\beta_{1}-a\right)}{k_{0}} \frac{d x^{\frac{k_{0}}{2}}(t)}{d t} .
\end{aligned}
$$

Finalmente, como $\lim _{t \rightarrow \infty} y(t)$ tem que existir, temos para o mesmo que

$$
\lim _{t \rightarrow \infty} y(t)<y\left(t_{0}\right)+\int_{t_{0}}^{\infty}-\frac{2\left(\beta_{1}-a\right)}{k_{0}} \frac{d x^{\frac{k_{0}}{2}}}{d t} d t=
$$




$$
\begin{aligned}
& =y\left(t_{0}\right)+\frac{2\left(\beta_{1}-a\right)}{k_{0}} x\left(t_{0}\right)^{\frac{k_{0}}{2}}-\lim _{t \rightarrow \infty} \frac{2\left(\beta_{1}-a\right)}{k_{0}} x(t)^{\frac{k_{0}}{2}}= \\
& =\left(1+\frac{2\left(\beta_{1}-a\right)}{k_{0}}\right) x\left(t_{0}\right)^{\frac{k_{0}}{2}}<0,
\end{aligned}
$$

o que é um absurdo, dada as considerações anteriores.

Teorema 2.9 Se $\beta_{1}>0$ e se $c=\min \left(2 k_{0}-1-k_{1}, k_{0}-k_{1}+l_{1}\right)$, então existem $S(x, y)$ e $R(x, y)$, tais que nenhuma solução de (2.3) assintótica à origem é tangente ao semi-eixo $\{(x, y) \mid y=0, x \geq 0\}$.

Prova: A idéia presente neste teorema é a mesma do teorema passado. Consideramos o sistema

$$
\begin{aligned}
& \dot{x}=-x^{k_{0}}+\alpha_{1} y x^{k_{1}} \\
& \dot{y}=\beta_{1} y x^{l_{1}}-a x^{c}
\end{aligned}
$$

em que, sem perda de generalidade, $\alpha_{1}>0$. Seja $a>\left|\frac{\beta_{1}}{\alpha_{1}}\right|+$ $\left|k_{0}-k_{1}\right|$.

Outra vez, se $\phi(t)=(x(t), y(t))$ é uma solução assintótica à origem tangente ao semi-eixo $x>0$, então $y(t)$ é positiva para todo $t \in \mathbf{R}$, pois se $y \leq 0$, então $\dot{y}<0$. Por outro lado, se $y>\frac{x^{k_{0}-k_{1}}}{\alpha_{1}}$, então $\dot{x}>0$.

Procedendo como no teorema passado, mostramos que existe $t_{0}$ para o qual $y(t)<\frac{x(t)^{k_{0}-k_{1}}}{\alpha_{1}}$, para todo $t \in\left[t_{0}, \infty\right)$. Além disso $\dot{x}>-x^{k_{0}}$ e então, para $t>t_{0}$, temos que

$$
\dot{y}<\left(\frac{\beta_{1}}{\alpha_{1}}-a\right) x^{c} \leq\left(\frac{\beta_{1}}{\alpha_{1}}-a\right) x^{2 k_{0}-1-k_{1}}<-\frac{\left(\frac{\beta_{1}}{\alpha_{1}}-a\right)}{k_{0-k_{1}}} \frac{d x^{k_{0}-k_{1}}}{d t} .
$$

Finalmente, observemos que

$\lim _{t \rightarrow \infty} y(t)<y\left(t_{0}\right)+\int_{t_{0}}^{\infty}-\frac{\left(\frac{\beta_{1}}{\alpha_{1}}-a\right)}{k_{0}-k_{1}} \frac{d x^{k_{0}-k_{1}}}{d t} d t=$ 


$$
\begin{aligned}
& =y\left(t_{0}\right)+\frac{\left(\frac{\beta_{1}}{\alpha_{1}}-a\right)}{k_{0}-k_{1}} x\left(t_{0}\right)^{k_{0}-k_{1}}-\lim _{t \rightarrow \infty} \frac{\left(\frac{\beta_{1}}{\alpha_{1}}-a\right)}{k_{0}-k_{1}} x(t)^{k_{0}-k_{1}}= \\
& =\left(1+\frac{\left(\frac{\beta_{1}}{\alpha_{1}}-a\right)}{k_{0}-k_{1}}\right) x\left(t_{0}\right)^{k_{0}-k_{1}}<0
\end{aligned}
$$

o que é absurdo, pois mostramos anteriormente que $y(t)$ era positiva para todo $t>t_{0}$.

Teorema 2.10 Se $c=2 k_{0}-k_{1}-1, l_{1}>k_{0}-1, \beta_{1}<0$, e $k_{1}<1$ podemos encontrar $S, R$ de tal forma que não exista solução de (2.3) assintótica à origem e tangente ao semi-eixo $x \geq 0$.

Prova: Analisaremos, sem perda de generalidade, apenas o caso em que $\alpha_{1}>0$. Começamos por analisar o sistema

$$
\begin{aligned}
& \dot{x}=-x^{k_{0}}+\alpha_{1} y x^{k_{1}} \\
& \dot{y}=\beta_{1} y x^{l_{1}}-a x^{c}-y^{2}
\end{aligned}
$$

onde $a>-\beta_{1}$, e admitiremos, por redução ao absurdo, que existe uma solução $\phi(t)=(x(t), y(t))$, trajetória assintótica à origem de (2.3).

Procedendo de maneira inteiramente análoga ao último teorema, temos que existe $\bar{t}_{0} \in \mathbf{R}$, tal que $y\left(\bar{t}_{0}\right)<0$. Então $y(t)<0$, para todo $t>\bar{t}_{0}$.

Notemos também que, se $-y<x^{c-l_{1}}$, segue-se que $\dot{y}<0$, e como $y\left(\bar{t}_{0}\right)<0$, tem de existir $\bar{t}_{1}>\bar{t}_{0}$ tal que $-y\left(\bar{t}_{1}\right)=x^{c-l_{1}}\left(\bar{t}_{1}\right)$.

Verifica-se facilmente que, se $t>\bar{t}_{1}$, então $-y(t)>x^{c-l_{1}}(t)$. Além disso, dado que $\phi(t)$ é assintótica, existe $\tilde{t}$ tal que $x(t)<1$, para todo $t>\tilde{t}$.

Consideramos agora a função

$$
V(x, y)=\frac{\ln (-y)}{\ln (x)}
$$


e calcularemos $\dot{V}(x(t), y(t))$, para $t>\max \left(\bar{t}_{1}, \tilde{t}\right)$.

Claramente, $V \leq c-l_{1}$. Mostraremos que $\liminf _{t \rightarrow \infty} V(x(t), y(t)) \leq$ 1 , o que é absurdo pois estamos supondo que $\phi$ é tangente ao eixo $y=0$.

Calculando, obtemos

$$
\dot{V}=\frac{\frac{\dot{y}}{y} \ln (x)-\frac{\dot{x}}{x} \ln (-y)}{\ln ^{2}(x)}=\frac{\frac{\dot{y}}{y}-\frac{\dot{x}}{x} V}{\ln (x)} .
$$

Por outro lado, se $y$ é negativo e $t$ é suficientemente grande, temos que

$$
\frac{\dot{y}}{y}>\beta_{1} x^{l_{1}}>\frac{-x^{k_{0}-1}}{2}>\frac{\dot{x}}{2 x}
$$

pois $l_{1}>k_{0}-1$.

Finalmente, se supusermos por absurdo que $V(t)>1$ em qualquer instante $t>\max \left(\bar{t}_{1}, \tilde{t}\right)$, teremos então que

$$
\dot{V}<\frac{\frac{\dot{x}}{x}}{2 \ln x}=-\frac{1}{2} \frac{d \ln (-\ln (x(t)))}{d t}<0,
$$

e portanto, para qualquer $t_{2}>\max \left(\bar{t}_{1}, \tilde{t}\right)$, temos

$$
\lim _{t \rightarrow \infty} V(t)<V\left(t_{2}\right)+\int_{t_{2}}^{\infty}-\frac{1}{2} \frac{d \ln (-\ln (x(t)))}{d t}=-\infty,
$$

o que claramente é absurdo, encerrando a demonstração.

Teorema 2.11 No sistema (2.3) suponha que $\beta_{1}<0, l_{1}<k_{0}-1$, $k_{1}<\frac{k_{0}}{2}$. Se $c \leq \max \left(1+l_{1}, k_{1}+l_{1}\right)$, então existe $a \in \mathbf{R}^{*}, a \alpha_{1}<0$, de forma que, se $S(x, y)=a x^{c}, R(x, y)=y^{2}$, não existe trajetória assintótica à origem, tangente a semi-reta $x \geq 0$. 
Prova: Suponhamos, sem perda de generalidade, que $\alpha_{1}<0$, e seja $a>\beta_{1} \alpha_{1}$.

Considere o sistema de equações

$$
\begin{aligned}
& \dot{x}=-x^{k_{0}}+\alpha_{1} y x^{k_{1}}+y^{2} \\
& \dot{y}=a x^{c}+\beta_{1} y x^{l_{1}},
\end{aligned}
$$

e seja, por hipótese de absurdo, $\phi(t)=(\bar{x}(t) ; \bar{y}(t))$ uma solução assintótica à origem, tangente ao semi-eixo $x \geq 0$.

Primeiro mostraremos que existe um instante $t_{0}$ para o qual $\phi\left(t_{0}\right)$ é positiva.

Temos que, na região onde $y<-\frac{1}{\alpha_{1}} x^{k_{0}-k_{1}}, \dot{x}$ é positivo. Logo, existe $t_{1}$ tal que $\bar{y}(t)>-\frac{1}{\alpha_{1}} \bar{x}(t)^{k_{0}-k_{1}}$, para todo $t>t_{1}$.

Como $c \leq \max \left(1+l_{1}, k_{1}+l_{1}\right)<k_{0}-k_{1}+l_{1}<2 k_{0}-k_{1}-1$, uma análise semelhante à realizada no Teorema 2.9 mostra que existe $t_{0}$ nas condições desejadas.

Temos também que, como a região $y>0$ é positivamente invariante, $\bar{y}(t)>0$, para todo $t>t_{0}$.

Notemos agora que, restrito à região onde $y<\frac{-a}{\beta_{1}} x^{c-l_{1}}, \dot{y}$ é positiva. Logo, a solução não pode, para $t$ suficientemente grande, ficar restrita a esta região.

Se $c \leq 1+l_{1}$, isto basta para mostrar que a solução não é tangente ao eixo dos $x$, o que é absurdo.

Se por outro lado $1+l_{1}<c \leq k_{1}+l_{1}$, temos que, restritos à região $y>\frac{-a}{\beta_{1}} x^{c-l_{1}}>-\alpha_{1} x^{c-l_{1}}$ para $x$ suficientemente pequeno, $\dot{x}>0$. Repetindo o mesmo argumento do Teorema 2.8, vemos que não existe trajetória assintótica à origem.

Teorema 2.12 No sistema (2.3) suponha que $\beta_{1}<0, l_{1}<k_{0}-1$ e que $k_{1}<1$. Então, se $c=2 l_{1}+1-k_{1}$, existe $a \in \mathbf{R}^{*}$, a $\alpha_{1}<$ 
0 , tal que, se $S(x, y)=a x^{c}$ e $R(x, y)=0$, não existe trajetória assintótica à origem, tangente à semi-reta $x \geq 0$.

Prova: Suponhamos, sem perda de generalidade, que $\alpha_{1}<0$, e seja $a>-2 \frac{\beta_{1}^{2}}{\alpha_{1}}$.

Considere o sistema de equações

$$
\begin{aligned}
& \dot{x}=-x^{k_{0}}+\alpha_{1} y x^{k_{1}} \\
& \dot{y}=a x^{c}+\beta_{1} y x^{l_{1}},
\end{aligned}
$$

e seja, por hipótese de absurdo, $\phi(t)=(\bar{x}(t) ; \bar{y}(t))$ uma solução assintótica à origem, tangente ao semi-eixo $x \geq 0$.

De maneira análoga ao resultado anterior, pode-se mostrar que existe um instante $t_{0}$ tal que $\phi\left(t_{0}\right)>0$. Temos também que, como a região $y>0$ é positivamente invariante, $\bar{y}(t)>0$, para todo $t>t_{0}$.

Notemos agora que na região onde $y<\frac{-a}{\beta_{1}} x^{c-l_{1}}=\frac{-a}{\beta_{1}} x^{l_{1}+1-k_{1}}$, $\dot{y}>0, \operatorname{logo}$ existe $t_{2}>t_{1}$ de forma que se $t>t_{2}$, então $\bar{y}(t)>$ $\frac{-a}{\beta_{1}} \bar{x}(t)^{l_{1}+1-k_{1}}$.

Finalmente, como estamos supondo que $\phi(t)$ é assintótica à origem, existe $t_{3}>t_{2}$ tal que, para $t>t_{3}, 0<\bar{x}(t)<1$.

Consideremos agora a função

$$
V(t)=\frac{\ln (\bar{y}(t))}{\ln (\bar{x}(t))}
$$

definida para $t>t_{3}$.

A derivada de $V$ com respeito ao tempo é

$$
\dot{V}=\frac{\frac{\dot{\bar{y}}}{\bar{y}} \ln (\bar{x}(t))-\frac{\dot{\bar{x}}}{\bar{x}} \ln (\bar{y}(t))}{\ln ^{2}(\bar{x}(t))}<\frac{\frac{\dot{\bar{y}}}{\bar{y}}-\frac{\dot{\bar{x}}}{\bar{x}} V}{\ln (\bar{x}(t))} .
$$


Como $\bar{y}(t)>\frac{-a}{\beta_{1}} \bar{x}(t)^{l_{1}+1-k_{1}}>2 \frac{\beta_{1}}{\alpha_{1}} \bar{x}(t)^{l_{1}+1-k_{1}}$, vale que

$$
\left|\frac{\frac{d \bar{y}}{d t}}{y}\right|<\beta_{1} x^{l_{1}}<\frac{1}{2}\left|\frac{\frac{d x}{d t}}{x}\right| .
$$

Se admitirmos por contradição que $V(t)>1$, para todo instante $t>t_{2}$, então teremos que

$$
\dot{V}<\frac{-1}{2} \frac{\frac{\frac{d x}{d t}}{x}}{\ln (\bar{x}(t))}=\frac{1}{2} \frac{d(\ln (-\ln (\bar{x}(t)))}{d t} .
$$

Como $\lim _{t \rightarrow \infty} \bar{x}(t)=0$, isto claramente implica em $\lim _{t \rightarrow \infty} V(t)=$ $-\infty$, o que é absurdo.

Teorema 2.13 No sistema (2.3) suponha que $\beta_{1}<0, l_{1} \leq k_{1}<$ 1. Então, se $c \leq k_{0}-k_{1}+l_{1}$, existe $a \in \mathbf{R}^{*}$, a $\alpha_{1}>0$, tal que, se $S(x, y)=a x^{c}, R(x, y)=0$, não existe trajetória assintótica à origem, tangente à semi-reta $x \geq 0$.

Prova: Suponhamos, sem perda de generalidade, que $\alpha_{1}>0$.

Seja $a>\frac{-\beta_{1}}{\alpha_{1}}$, e suponha por absurdo que existe uma solução $\phi(t)=(\bar{x}(t), \bar{y}(t))$, assintótica à origem, tangente ao semi-eixo $x \geq 0$.

Nosso sistema tem equações

$$
\begin{aligned}
& \dot{x}=-x^{k_{0}}+\alpha_{1} y x^{k_{1}} \\
& \dot{y}=a x^{c}+\beta_{1} y x^{l_{1}} .
\end{aligned}
$$

Visto que $c \leq k_{0}$, restrita ao quadrante $y \leq 0, x \geq 0$, a função

$$
\frac{-\dot{x}}{\dot{y}}=\frac{x^{k_{0}}-\alpha_{1} y x^{k_{1}}}{a x^{c}+\beta_{1} y x^{l_{1}}},
$$


é estritamente menor do que uma constante positiva. Isso mostra que toda solução, quando restrita a este quadrante, tem uma inclinação mínima com relação ao semi-eixo $x \geq 0$.

Portanto, qualquer solução que seja assintótica ao mesmo semieixo (e em particular $\phi(t))$ tem que, em um dado instante $t_{0}$, estar na região $y>0$.

Além disso, como esta região é invariante, visto que $\dot{y} \geq 0$ sobre o eixo $y=0$, vale que para todo $t \in\left(t_{0}, \infty\right), \bar{y}(t)$ é positiva.

Notemos também que, se

$$
0<\bar{y}(t) \leq \frac{1}{\alpha_{1}} \bar{x}^{k_{0}-k_{1}} \leq \frac{a}{-\beta_{1}} \bar{x}^{c-l_{1}},
$$

então $\bar{y}(t)$ é crescente.

Logo, como $\phi$ é assintótica, existe um instante $t_{1}$ tal que $\bar{y}\left(t_{1}\right)=$ $\frac{1}{\alpha_{1}} \bar{x}^{k_{0}-k_{1}}\left(t_{1}\right)$.

Ainda, se $\frac{1}{\alpha_{1}} \bar{x}^{k_{0}-k_{1}} \leq \bar{y}(t)$, então $\bar{x}(t)$ é crescente.

Portanto, a região $K=\left\{(x, y) \mid x>\frac{1}{\alpha-1} \bar{x}^{k_{0}-k_{1}}\left(t_{1}\right), y>\bar{y}\left(t_{1}\right)\right\}$ é positivamente invariante, e a solução $\phi(t)$ tem de permanecer na mesma para qualquer $t>t_{1}$, o que contradiz o fato de $\phi$ ser assintótica à origem.

Teorema 2.14 No sistema (2.3) suponha que $\beta_{1}<0, k_{1}<1, k_{1}<$ $l_{1}<k_{0}-1$. Então, se $c \leq 1+k_{1}$, existe $a \in \mathbf{R}^{*}$, a $\alpha_{1}>0$, tal que, se $S(x, y)=a x^{c}, R(x, y)=0$, não existe trajetória assintótica à origem, tangente à semi-reta $x \geq 0$.

Prova: Vamos admitir que $\alpha_{1}>0$. Considere o sistema

$$
\begin{aligned}
& \dot{x}=-x^{k_{0}}+\alpha_{1} y x^{k_{1}} \\
& \dot{y}=x^{c}+\beta_{1} y x^{l_{1}} .
\end{aligned}
$$

Veja que, se $|x|$ for menor que $\delta$ suficientemente pequeno, e se $|y|<\min \left(\frac{1}{3 \alpha_{1}}, \frac{-1}{3 \beta_{1}}\right) x$, então $\dot{y}>|\dot{x}|$, o que impede que trajetórias assintóticas à origem sejam tangentes à semireta $x \geq 0$. 
Teorema 2.15 No sistema (2.3) suponha que $\beta_{1}<0,1 \leq k_{1}, k_{1}<$ $l_{1}<k_{0}-1$. Então, se $c \leq k_{0}-k_{1}+l_{1}$, existe $a \in \mathbf{R}^{*}$, $a \alpha_{1}>0$, tal que, se $S(x, y)=a x^{c}, R(x, y)=0$, não existe trajetória assintótica à origem, tangente à semi-reta $x \geq 0$.

Prova: Admitiremos que $a>0$. Considere o sistema

$$
\begin{aligned}
& \dot{x}=-x^{k_{0}}+\alpha_{1} y x^{k_{1}} \\
& \dot{y}=a x^{c}+\beta_{1} y x^{l_{1}}+\left(k_{0}-1\right) 2 \alpha_{1} y^{2},
\end{aligned}
$$

onde $a>\frac{-\beta_{1}}{\alpha_{1}}$.

Suponhamos, por absurdo, que exista uma solução $\phi(t)=$ $(\bar{x}(t), \bar{y}(t))$ assintótica à origem, tangente ao semi-eixo $x \geq 0$.

Como no primeiro quadrante $\dot{y} \geq 0$, é claro que $\bar{y}(t)<0$ para todo $t \in \mathbf{R}$. Além disso, como $\phi(t)$ é tangente à $x \geq 0$ e assintótica à origem, temos que existe $t_{0}$ tal que, se $t>t_{0}, \bar{y}(t)<-\bar{x}(t) \mathrm{e}$ $0<\bar{x}(t)<1$.

Considere a função

$$
V(t)=\frac{\ln (-\bar{y}(t))}{\ln (\bar{x}(t))}
$$

definida para $t>t_{0}$

Afirmamos que existe um instante $t_{1}$ tal que $V\left(t_{1}\right)>k_{0}-1$. Para tanto, veja que se $V(t) \leq k_{0}-1$, então

$$
\dot{V}(t)=\frac{\dot{\bar{y}} \ln (\bar{x})-\frac{\dot{\bar{x}}}{\bar{x}} \ln (-\bar{y})}{\ln ^{2}(\bar{x})}=\left(\frac{\frac{\dot{y}}{\bar{y} V}-\frac{\dot{\bar{x}}}{\bar{x}}}{\ln (-\bar{y})}\right) V^{2} .
$$

Por outro lado, como $\phi(t)$ é assintótica à origem, existe $t_{2}$ tal que, se $t>t_{2},\left|\bar{x}^{k_{0}-1}(t)\right|<2\left(k_{0}-1\right)\left|\beta_{1} \bar{x}^{l_{1}}(t)\right|$.

Logo, se $t>t_{2}$ e $V(t) \leq k_{0}-1$ um cálculo nos mostra que

$$
\dot{V}(t)>\left(\frac{\frac{\dot{\bar{y}}}{\bar{y}}}{\ln (-\bar{y})}\right) \frac{V}{2}>\frac{1}{2} \frac{d \ln (-\ln (-\bar{y})}{d t},
$$


e portanto

$$
\lim _{t \rightarrow \infty} V(t)>V\left(t_{2}\right)+\int_{t_{2}}^{\infty} \frac{1}{2} \frac{d \ln (-\ln (-\bar{y})}{d t} d t=+\infty .
$$

Isto implica na existência do instante $t_{1}$ tal que, para $t>t_{1}$, $\bar{y}(t)>-\bar{x}^{k_{0}-1}(t)$. Finalmente, uma análise semelhante à realizada no Teorema 2.9 mostra que existe $t_{3}>0$ onde $\bar{y}\left(t_{3}\right)>0$, obtendo outra vez absurdo e completando a demonstração.

O leitor poderá verificar que os resultados 2.7 à 2.15 mostram que, exceto no caso em que $\beta_{1}<0$ e $l_{1}=k_{0}-1$, os Teoremas 2.4, 2.5 e 2.6 fornecem as melhores estimativas possíveis para $c$.

\subsection{Estudo de trajetórias assintóticas em três dimensões}

Terminado o estudo realizado na última seção, uma questão pertinente é como os resultados obtidos podem ser generalizados, e quais aspectos do problema são intrínsicos ao caso bi-dimensional.

Nesta seção estudaremos o caso tri-dimensional e veremos que, com hipóteses "semelhantes" a hiperbolicidade, os resultados da seção anterior se estendem naturalmente. Entretanto, na ausência de tais hipóteses comportamentos consideravelmente mais complexos podem aparecer.

Começaremos por colocar o problema de um modo mais preciso, tentando manter o mesmo espírito da seção anterior, e estudaremos sistemas de equações do tipo

$$
\begin{aligned}
\dot{x} & =-x^{k_{0}}+\alpha_{1} y x^{k_{1}}+\gamma_{1} z x^{k_{2}}+R(x, y, z) \\
\left(\begin{array}{l}
\dot{y} \\
\dot{z}
\end{array}\right) & =A\left(\begin{array}{l}
y \\
z
\end{array}\right) x^{l_{1}}+\left(\begin{array}{l}
S(x, y, z) \\
T(x, y, z)
\end{array}\right)
\end{aligned}
$$


onde $R \in O\left(y^{2}\right)+O\left(z^{2}\right)+O(z y)+o\left(x^{k_{0}}\right)+o\left(z x^{k_{2}}\right)+o\left(y x^{k_{1}}\right)$, $S, T \in O\left(y^{2}\right)+O\left(z^{2}\right)+O(z y)+O\left(x^{c}\right)+o\left(z x^{l_{1}}\right)+o\left(y x^{l_{1}}\right)$, e $A=\left[\begin{array}{ll}a_{11} & a_{12} \\ a_{21} & a_{22}\end{array}\right]$ é uma matriz real dois por dois com coeficientes constantes.

Outra vez estaremos interessados em, conhecidos os valores de $k_{0}, k_{1}, k_{2}, \alpha_{1}, \gamma_{1}, l_{1}$ e a matriz $A$, encontrar condições sobre $c$ que assegurem a existência de trajetórias assintóticas à origem tangentes à semi-reta $x \geq 0$. Também aqui suporemos que $k_{0} \geq 2$, e que $k_{1}, k_{2}$, e $l_{1}$ não são negativos.

Uma inspeção do problema bidimensional tratado mostra que, em (2.24), $A$ desempenha o mesmo papel que $\beta_{1}$ em (2.3), mas a matriz $A$ terá uma influência mais sensível do que aquela observada para $\beta_{1}$.

Começaremos com os resultados positivos, do tipo do Teorema 2.4

Teorema 2.16 Se $l_{1}>k_{0}-1$, e se

$$
c>\tilde{c}=\max \left(2 k_{0}-2,2 k_{0}-1-k_{1}, 2 k_{0}-1-k_{2}\right),
$$

então existe uma trajetória assintótica à origem tangente ao semieixo $x \geq 0$.

Prova: A demonstração é muito similar àquela do Teorema 2.4. Começamos por tomar duas funções, definidas para $x>0$ por

$$
\begin{aligned}
V_{1}(x, y, z) & =x, \\
V_{2}(x, y, z) & =\frac{y^{2}+z^{2}}{x^{2 d}},
\end{aligned}
$$

onde $d=\max \left(k_{0}-1, k_{0}-k_{1}, k_{0}-k_{2}\right)+\frac{c-\tilde{c}}{2}$.

Verificaremos que existe $\delta$ real positivo suficientemente pequeno tal que em $\Omega_{1}=\left\{(x, y, z) \mid 0<x<\delta, V_{2}(x, y, z) \leq 1\right\}$, vale 
que $\dot{V}_{1}<0$ e em $B=\left\{(x, y, z) \mid 0<x<\delta, V_{2}(x, y, z)=1\right\}$, temos que $\dot{V}_{2}>0$.

Para tanto, note que

$$
\dot{V}_{2}=\frac{(2 y \dot{y}+2 z \dot{z}) x-2 d \dot{x}\left(y^{2}+z^{2}\right)}{x^{2 d+1}}
$$

e veja que, restrito a $V_{2}^{-1}(\varepsilon)$, temos

$$
\begin{aligned}
(2 y \dot{y}+2 z \dot{z}) x= & O\left(y^{2}+z^{2}\right) x^{l_{1}+1}+(O(y)+O(z)) x^{c+1}+ \\
& +\left(O\left(y^{3}\right)+O\left(z^{3}\right)\right) x= \\
= & O\left(x^{2 d+l_{1}+1}\right)+O\left(x^{d+c+1}\right)+O\left(x^{3 d}\right) . \\
2 d \dot{x}\left(y^{2}+z^{2}\right)= & 2 d\left(y^{2}+z^{2}\right)\left(-x^{k_{0}}+\alpha_{1} y x^{k_{1}}+\gamma_{1} z x^{k_{2}}+\right. \\
& +R(x, y, z))= \\
= & -2 d x^{k_{0}+2 d}+O\left(x^{k_{0}+2 d}\right)+O\left(x^{k_{1}+3 d}\right)+ \\
& +O\left(x^{k_{2}+3 d}\right)+O\left(x^{4 d}\right) .
\end{aligned}
$$

Logo, segue da definição de $d$ e da desigualdade $c>d+k_{0}-1$ que, para $x<\delta$ suficientemente pequeno,

$$
\dot{V}_{2}<-d x^{k_{0}-1} \text {. }
$$

Finalmente, temos que, restrita a $\Omega_{1}$,

$$
\dot{x}=-x^{k_{0}}+O\left(x^{k_{1}+d}\right)+O\left(x^{k_{2}+d}\right)+O\left(x^{2 d}\right)<0,
$$

o que, após uma aplicação direta do Teorema 2.2, prova o resultado.

Teorema 2.17 Se $l_{1}<k_{0}-1, A=\left[\begin{array}{cc}a_{11} & a_{12} \\ 0 & a_{22}\end{array}\right]$, onde $a_{11} a_{22} \neq$ 0 , e se $c>\tilde{c}=\max \left(2 l_{1}, k_{0}+l_{1}-k_{1}, k_{0}+l_{1}-k_{2}\right)$, então existe uma trajetória assintótica para a origem, tangente ao semi-eixo $x \geq 0$. 
Prova: Seja $d=\max \left(k_{0}-k_{1}, k_{0}-k_{2}, \frac{k_{0}}{2}, l_{1}\right)+\frac{c-\tilde{c}}{2}$. Considere as seguintes três funções, definidas em $\Omega_{2}=\{(x, y, z) \mid 0<x<\delta\}$, onde $\delta>0$ é um número suficientemente pequeno que será determinado mais tarde:

$$
\begin{aligned}
V_{1}(x, y, z) & =x \\
U_{2}(x, y, z) & =\frac{y^{2}}{x^{2 d}}, \\
U_{3}(x, y, z) & =\frac{z^{2}}{x^{2 d}},
\end{aligned}
$$

e seja

$$
\Omega_{1}=\left\{(x, y, z)\left|0<x<\delta, U_{2}(x, y, z) \leq 1, U_{3}(x, y, z) \leq\right| \frac{a_{11}}{2 a_{12}} \mid\right\},
$$
se $a_{12} \neq 0$, ou

$$
\Omega_{1}=\left\{(x, y, z) \mid 0<x<\delta, U_{2}(x, y, z) \leq 1, U_{3}(x, y, z) \leq 1\right\}
$$

caso contrário.

Calculando

$$
\begin{aligned}
\dot{V}_{1} & =\dot{x} \\
\dot{U}_{2} & =\frac{2 y \dot{y} x-2 d \dot{x} y^{2}}{x^{2 d+1}}, \\
\dot{U}_{3} & =\frac{2 z \dot{z} x-2 d \dot{x} z^{2}}{x^{2 d+1}}
\end{aligned}
$$

temos que, se $\delta$ for suficientemente pequeno, então $\dot{V}_{1}(x, y, z)<0$, para todo $(x, y, z) \in \Omega_{1}$, e nesta região temos também que $\dot{V}_{1}=$ $O\left(x^{k_{0}}\right)$.

Da mesma forma, se nos restringirmos a $\Omega_{1} \cap U_{2}^{-1}(1)$, teremos

$$
\begin{array}{r}
\dot{U}_{2}=\frac{2}{x^{2 d+1}}\left[a_{11} y^{2} x^{l_{1}+1}+a_{12} y z x^{l_{1}+1}+y^{2} O\left(x^{k_{0}}\right)+\right. \\
\left.+O\left(y^{3}+y z^{2}+y^{2} z\right) x+y O\left(x^{c+1}\right)+\text { tos }\right]
\end{array}
$$

e como nesta região, se $a_{12} \neq 0$, temos que $|z|<\left|\frac{a_{11}}{2 a_{12}} y\right|$, fica claro 
que, eventualmente diminuindo um pouco $\delta$, o sinal de $\dot{U}_{2}$ será o mesmo de $a_{11}$.

De maneira inteiramente análoga, podemos calcular $\dot{U}_{3}$ restrita a $\Omega_{1} \cap U_{3}^{-1}(1)$, quando $a_{12}=0$, ou restrita a $\Omega_{1} \cap U_{3}^{-1}\left(\left|\frac{a_{11}}{2 a_{12}}\right|\right)$ se $a_{12} \neq 0$. O leitor não terá dificuldades em verificar que nesta região o sinal de $\dot{U}_{3}$ não muda, e é o mesmo de $a_{22}$. Temos agora quatro possibilidades, a saber

1. $a_{11}>0, a_{22}>0$

2. $a_{11}>0, a_{22}<0$;

3. $a_{11}<0, a_{22}>0$;

4. $a_{11}<0, a_{22}<0$.

No primeiro caso, utilizamos o Teorema 2.2, tomando $V_{2}=$ $\max \left(U_{2}, U_{3}\right)$.

No segundo caso, utilizamos o Teorema 2.3, fazendo

$$
\begin{aligned}
V_{2} & =U_{3}, V_{3}=U_{2}, \\
C & =\left\{(x, y, z) \in \partial \Omega_{1} \mid y=-x^{d}\right\} \\
D & =\left\{(x, y, z) \in \partial \Omega_{1} \mid y=x^{d}\right\} .
\end{aligned}
$$

No terceiro caso, utilizamos o Teorema 2.3, com

$$
V_{2}=U_{2}, V_{3}=U_{3}
$$

$\mathrm{e}$, se $a_{12}=0$

$$
\begin{aligned}
& C=\left\{(x, y, z) \in \partial \Omega_{1} \mid z=-x^{d}\right\} \\
& D=\left\{(x, y, z) \in \partial \Omega_{1} \mid z=x^{d}\right\},
\end{aligned}
$$


ou se $a_{12} \neq 0$,

$$
\begin{aligned}
& C=\left\{(x, y, z) \in \partial \Omega_{1}|z=-| \frac{a_{11}}{2 a_{12}} \mid x^{d}\right\} \\
& D=\left\{(x, y, z) \in \partial \Omega_{1}|z=| \frac{a_{11}}{2 a_{12}} \mid x^{d}\right\} .
\end{aligned}
$$

Finalmente, no quarto caso, utilizamos o Teorema $2.1 \operatorname{com} V_{2}=$ $\max \left(U_{2}, U_{3}\right)$.

Portanto, todos os casos foram cobertos e, como cada um dos teoremas citados garantia a existência de uma trajetória assintótica, só restaria provar a tangência da mesma ao eixo dos $x$. Mas veja que isto já está garantido pelo simples fato de que $d>1$, o que termina a demonstração.

Teorema 2.18 Se $l_{1}<k_{0}-1, A=\left[\begin{array}{cc}a_{11} & a_{12} \\ -a_{12} & a_{11}\end{array}\right]$, onde $a_{11} \neq 0$, $e$

$$
c>\max \left(2 l_{1}, k_{0}+l_{1}-k_{1}, k_{0}+l_{1}-k_{2}\right),
$$

então existe uma trajetória assintótica para a origem, tangente ao semi-eixo $x \geq 0$.

Esboço da Prova: A demonstração deste teorema é muito similar a do teorema passado, portanto vamos apenas indicá-la.

Primeiramente, tomamos como funções auxiliares

$$
\begin{aligned}
V_{1}(x, y, z) & =x, \\
V_{2}(x, y, z) & =\frac{y^{2}+z^{2}}{x^{d}}
\end{aligned}
$$

onde $d$ é tomado como no último teorema.

Definimos $\Omega_{1}=\left\{(x, y, z) \mid 0<x<\delta, V_{2}=1\right\}$, onde $\delta$ outra vez será tomado suficientemente pequeno. 
Verificamos que $\dot{x}<0$ em $\Omega_{1}$, e se calcularmos

$$
\begin{aligned}
\dot{V}_{2} & =\frac{(2 y \dot{y}+2 z \dot{z}) x-\dot{x}\left(y^{2}+z^{2}\right)}{x^{2 d+1}}= \\
& =\frac{\left(y^{2}+z^{2}\right)\left(2 a_{11} x^{l_{1}+1}-\dot{x}\right)+H(x, y, z)}{x^{2 d+1}},
\end{aligned}
$$

veremos que, restrita a $(x, y, z) \in \Omega_{1}, V_{2}=1$, temos que o termo de ordem dominante é $\left(y^{2}+z^{2}\right) 2 a_{11} x^{l_{1}-2 d}$, cujo sinal nesta região, quando $\delta$ é suficientemente pequeno, é o mesmo de $a_{11}$. Prosseguimos então, aplicando o Teorema 2.1 caso $a_{11}<0$, ou aplicando o Teorema 2.2 caso $a_{11}>0$.

Finalmente, a tangência ao eixo $x$ é obtida observando que $d>1$.

Vamos agora apresentar um exemplo onde não existe trajetória assintótica, apesar da perturbação ser relativamente fraca (i.e., o valor de $c$ é relativamente grande).

Exemplo 2.1 Considere o sistema

$$
\begin{aligned}
& \dot{x}=-x^{k_{0}} \\
& \dot{y}=y^{2}+x^{c} \\
& \dot{z}=-y+z^{2}
\end{aligned}
$$

onde $c<3 k_{0}-3$. Então não existe trajetória assintótica à origem tangente ao semi-eixo $x \geq 0$.

Esboço de prova: Suponhamos, por absurdo, que existe $\phi(t)=$ $(x(t), y(t), z(t))$, solução de (2.35) assintótica à origem tangente ao semi-eixo $x \geq 0$. Notemos que, como $\dot{y}>0$, temos que $y(t)<0$ para todo $t \in \mathbf{R}$. Além disso, sabemos calcular

$$
x(t)=\left(x(0)+\left(k_{0}-1\right) t\right)^{\frac{-1}{k_{0}-1}} .
$$


Logo, visto que

$$
\dot{y}>x^{c}=-\frac{1}{\left(c-k_{0}+1\right)} \frac{d}{d t} x^{c-k_{0}+1},
$$

temos também que

$$
\begin{aligned}
\lim _{t \rightarrow \infty} y(t) & =0 \geq y(a)+\int_{a}^{\infty}-\frac{1}{\left(c-k_{0}+1\right)} \frac{d}{d t} x^{c-k_{0}+1}(s) d s= \\
& =y(a)+\frac{x^{c-k_{0}+1}(a)}{c-k_{0}+1}, \forall a \in \mathbf{R} .
\end{aligned}
$$

Esta última equação mostra que $y(t)<-\frac{x^{c-k_{0}+1}(t)}{c-k_{0}+1}$ para todo $t$ e, portanto, temos que

$$
\dot{z}>\frac{x^{c-k_{0}+1}(t)}{c-k_{0}+1}+z^{2} .
$$

Deste ponto em diante, a análise é idêntica àquela realizada no Teorema 2.7, que mostrava, quando $c-k_{0}+1<2 k_{0}-2$, não existir trajetória assintótica à origem tangente ao eixo dos $x$.

Uma comparação entre este exemplo e o Corolário 2.1 evidencia que sistemas de equações em dimensão maior do que dois possuem "patologias" próprias, com comportamentos intrinsecamente distintos daqueles observados nos casos bidimensionais. Tais "patologias" não são tão simples de serem tratadas ou compreendidas, e requerem um estudo posterior mais aprofundado.

Como um último comentário, gostaríamos de apontar que a demonstração do teorema 2.16 pode ser estendida trivialmente para o caso $n+1$-dimensional, feitas algumas adptações óbvias nas hipóteses do teorema como substituir na equação $(2.24)(y, z)$ por $n$ variáveis e transformando a matriz $A$ numa matriz $n \times n$. 


\section{Capítulo 3 \\ A Inversão Do Teorema De Dirichlet- Lagrange}

\subsection{Introdução}

Neste Capítulo estudaremos a estabilidade de pontos de equilíbrio de sistemas lagrangeanos com dois graus de liberdade. Trataremos especialmente do caso em que o jato de ordem $k$ da energia potencial na origem mostra que $\mathbf{0}$ não é um ponto de mínimo da mesma. Mostraremos nesta situação que, sob hipóteses mínimas de regularidade das energias potencial e cinética, existe uma solução das equações de Lagrange que é assintótica à origem no passado, e portanto $(\mathbf{0}, \mathbf{0})$ é um equilíbrio instável.

Para evitar complicações desnecessárias, sempre que nos referirmos ao jato $k$ de uma função $f$, ficará subentendido que o mesmo é o jato de ordem $k$ da função na origem, e o denotaremos por $j^{k} f$.

Precisamos apresentar uma definição mais precisa da frase "O jato de ordem $k$ de $f$ mostra que 0 não é um ponto de mínimo da mesma." Este é um conceito intimamente relacionado com o conceito de $k$-decidibilidade, que será discutido em mais detalhes no apêndice. Portanto, a similaridade entre a próxima definição e 
a Definição 5.1 não é simples coincidência.

Definição 3.1 Diremos que o jato $k$ de uma função $f$ mostra que a mesma não tem mínimo na origem se, para toda função $g$ tal que $j^{k} g=j^{k} f$, 0 não é um ponto de mínimo de $g$.

Por último, gostaríamos de relembrar o resultado de [BGZ] que prova que, se uma função analítica $f$ não tem mínimo, nem mesmo brando, na origem, então existe um número natural $k$ tal que $j^{k} f$ mostra que a mesma não tem mínimo em $\mathbf{0}$.

Isto bastará para comprovar que o nosso resultado é uma extensão para dois graus de liberdade, com menos restrições de regularidade, dos resultados já citados de Palamodov e Moauro-Negrini (Teoremas (1.8) e (1.11) do Capítulo 1).

\subsection{O Problema}

Consideremos um sistema lagrangeano conservativo com dois graus de liberdade, de energia potencial $\pi$ definida na vizinhança aberta da origem $\Omega$ e energia cinética $T$ definida em $\mathbf{R}^{2} \times \Omega$.

Admitiremos que $\pi$ é de classe $\mathcal{C}^{2}, \pi(0)=\|\pi(0)\|=0$ e que $\pi=\mathrm{P}+\mathrm{R}$, onde $\mathrm{P}$ é um polinômio de grau menor ou igual a $k \mathrm{e}$ $\lim _{x \rightarrow 0} \frac{\pi(x)-\mathrm{P}(x)}{\|x\|^{k}}=0$, ou seja, $\mathrm{P}$ é o jato $k$ de $\pi$ na origem. Suporemos também que:

- P mostra que $\pi$ não tem mínimo na origem.

- Existe o jato $k-1$ do gradiente de $\pi$.

- Os jatos de ordens menores do que $k$ não mostram que $\pi$ não tem mínimo na origem. 
Vale lembrar que a segunda hipótese basta para demonstrar o seguinte resultado simples, que utilizaremos no restante do trabalho.

Fato 3.1 Se fé uma função de classe $\mathcal{C}^{2}$, definida num aberto $\Omega$ que contém a origem, e existe o jato de ordem $k-1$ do gradiente de $f$, então também existe $j^{k} f$ e vale que:

$$
\nabla j^{k} f=j^{k-1} \nabla f
$$

\section{Prova: Veja [Gar-M]}

Nossa energia cinética é uma forma quadrática definida positiva nas velocidades e será suposta de classe $\mathcal{C}^{2}$, isto é,

$$
\mathrm{T}=\frac{1}{2}\langle\mathrm{~B}(q) \dot{q} \mid \dot{q}\rangle
$$

onde $\mathrm{B}(q)=\left[\begin{array}{ll}\mathrm{F}(q) & \mathrm{G}(q) \\ \mathrm{G}(q) & \mathrm{H}(q)\end{array}\right]$ é definida positiva para todo $q \in \Omega$ e $T, G$ e $H$ são funções de classe $\mathcal{C}^{2}$ definidas em $\Omega$. Sem perda de generalidade, faremos a hipótese $\mathrm{B}(0)=\mathrm{I}$, o que poderia ser obtido com uma simples mudança linear de coordenadas.

Nestas condições $(0 ; 0)$ é um ponto de equilíbrio das equações de Lagrange do sistema de lagrangeana $L=T-\pi$. O que pretendemos é provar que as hipóteses feitas acima garantem a instabilidade de Liapounof desse equilíbrio.

O seguinte resultado de $k$-decidibilidade está demonstrado no Apêndice, desempenhando um papel significativo em nosso trabalho.

Fato 3.2 Se o jato $k$ de $f$ na origem mostra que esta função não tem minimo em 0 , e se os jatos de ordem menor do que $k$ não 
mostram que $f$ não tem mínimo em $\mathbf{0}$, então existem, eventualmente após uma rotação em $\mathbf{R}^{2}$, reais $\lambda>0, \alpha>0$ e uma curva algébrica $\Gamma(x)=(x ; \gamma(x))$, onde $\gamma:[0 ; \varrho[\longrightarrow \mathbf{R}, \operatorname{com} \Gamma(0)=0 e$ cujo versor em $0+$ é $(1 ; 0)$, tais que

$$
\min _{-\lambda x<y<\lambda x} \mathrm{P}(x ; y)=\mathrm{P}(x ; \gamma(x))=-\alpha x^{\bar{\beta}}+o\left(\|x\|^{\bar{\beta}}\right), \forall x \in[0 ; \varrho[,
$$

$\operatorname{com} \bar{\beta} \leq k$.

Observe que como $\Gamma$ é algébrica nas condições desta proposição, podemos admitir sem perda de generalidade que (veja o texto de Walker[W] sobre curvas algébricas, para uma apresentação elegante e completa do Teorema de Puiseaux que mostra este resultado)

$$
\gamma(x)=\sum_{j=1}^{+\infty} b_{j} x^{\beta_{j}}
$$

com $b_{j} \in \mathbf{R}$ e $\left(\beta_{j}\right)$ é uma seqüência de racionais estritamente crescente com $\beta_{1}>1$. Além disso, exceto no caso em que $\Gamma$ coincide com o semi-eixo das abscissas (situação em que $\gamma \equiv 0$ ) tem-se que a série dada em (3.3) é única, $\operatorname{com} \beta_{1}$ diferente de zero.

Resultam imediatamente destas observações que, eventualmente diminuindo um pouco $\varrho$, existem constantes $c_{1}, c_{2}$ e $c_{3}$ positivas tais que, para $0<x<\varrho$

$$
\begin{array}{r}
|\gamma(x)|<c_{1} x^{\beta_{1}} \\
\left|\gamma^{\prime}(x)\right|<c_{2} x^{\beta_{1}-1} \\
\left|\gamma^{\prime \prime}(x)\right|<c_{3} x^{\beta_{1}-2} .
\end{array}
$$

Destacamos também que como $\beta_{1}>1$ tem-se $\beta_{1}-2>-1$.

Estas propriedades e estimativas acerca de $\Gamma$ serão usadas nas seções seguintes. 


\subsection{Um Lema Fundamental}

Nesta seção provaremos um resultado fundamental que nos permitirá estabelecer a existência de uma trajetória assintótica para a origem nas hipóteses colocadas na seção anterior.

Lembremos que $\pi: \Omega \longrightarrow \mathrm{R}$ é uma função de classe $\mathcal{C}^{2}, \pi(0)=$ $\|\nabla \pi(0)\|=0$, e $\pi$ tem jato $k$ na origem e esse jato mostra que $\pi$ não tem mínimo em 0 .

Tomemos $\mathrm{P}=j^{k} \pi$ e consideremos a curva $\Gamma(x)=(x, \gamma(x))$ mensionada na seção anterior que satisfaz o Fato 3.2. Uma vez que $\Gamma$ obedece (3.2) iremos denominá-la curva de mínimos vertical de $\mathrm{P}$.

Comecemos fazendo uma mudança de coordenadas de classe $\mathcal{C}^{\infty}$ na faixa vertical $\mathcal{F}=\{(x ; y): 0<x<\varrho\}$ que admitirá uma extensão trivial à faixa $\overline{\mathcal{F}}=\{(x: y): x<\varrho\}$ que será um difeomorfismo de classe $\mathcal{C}^{1}$. O objetivo dessa mudança será transformar a curva $\Gamma$ no segmento $\{(x ; 0), 0<x<\varrho\}$.

Consideremos

$$
\varphi(x ; y)=(x ; w)= \begin{cases}(x ; y-\gamma(x)), & \text { se } 0<x<\varrho \\ (x ; y), & \text { se } x \leq 0\end{cases}
$$

Em virtude de (3.3) e das observações feitas ao final da seção 1 , é claro que $\varphi$ é um difeomorfismo de classe $\mathcal{C}^{1}$ (pois $\beta_{1}>1$, aliás, $\varphi$ tem classe $\left.\left\lfloor\beta_{1}\right\rfloor\right)$ de $\mathbf{R}^{2}$ em si mesmo.

Deste ponto em diante, trabalharemos com as coordenadas $(x ; w)$, tomando o cuidado de nos restringirmos à faixa $\mathcal{F}$, onde a nossa mudança de coordenadas tinha classe $\mathcal{C}^{\infty}$. No futuro, nos bastará lembrar que $\varphi$ tem uma extensão à faixa $\overline{\mathcal{F}}$ que é um difeomorfismo.

Escreveremos agora $\mathrm{P}$ nas coordenadas $(x ; w)$. Como $\mathrm{P}$ é um polinômio de grau menor ou igual a $k$, temos $\mathrm{P}(x ; y)=\sum_{(i ; j) \in I} a_{i j} x^{i} y^{j}$, 
portanto, da definição de $\varphi$ e de (3.3) resulta, por substituição direta,

$$
\mathrm{P}(x ; w)=\sum_{(i, j) \in I} \sum_{k=0}^{j} a_{i j} x^{i}\left(\frac{j}{k}\right)\left(\sum_{\ell=1}^{\infty} b_{\ell} x^{\beta_{\ell}}\right)^{k-j} w^{k}
$$

Observemos que $\varphi$ deixa invariantes as retas verticais $x=\xi$ e, em cada uma dessas retas, é uma translação $(w=y+\gamma(\xi))$. Lembrando ainda que $\varphi$ leva a curva $\Gamma$ no eixo dos $x$, resulta de (3.2) que:

(i) Nas coordenadas $(x ; w), \mathrm{P}(x ; 0)=-\alpha x^{\bar{\beta}}$.

(ii) Para $\xi \in(0, \varrho)$ fixo, a função $\ell(w)=\mathrm{P}(\xi ; w), w \in \mathbf{R}$, tem um ponto de mínimo local em 0 .

De (ii) resulta que $\frac{\partial \mathrm{P}}{\partial w}(x ; 0)=0,0<x<\varrho$ e portanto na expressão (3.5) não existem termos lineares em $w$.

Separando os termos de (3.5) com grau menor ou igual a $\bar{\beta}$ e observando que os expoentes de $w$ são todos naturais maiores ou iguais a dois e os expoentes de $x$ são racionais maiores ou iguais a 1, temos:

$$
\mathrm{P}(x ; w)=-\alpha x^{\bar{\beta}}+w^{2} \sum_{i \in \mathbf{Q}, j \in \mathbf{N}}^{i+j \leq \bar{\beta}} \widetilde{a}_{i j} x^{i} w^{j-2}+o\left(\|x ; w\|^{\bar{\beta}}\right),
$$

assim, fazendo $P_{2}(x, w)=w^{2} \sum_{i \in \mathbf{Q}, j \in \mathbf{N}}^{i+j \leq \bar{\beta}} \widetilde{a}_{i j} x^{i} w^{j-2}$, vem (note que $\widetilde{a}_{i j} \neq 0$ em apenas um subconjunto finito)

$$
\mathrm{P}(x ; w)=-\alpha x^{\bar{\beta}}+P_{2}(x, w)+o\left(\|x ; w\|^{\bar{\beta}}\right) .
$$

Um cálculo imediato mostra que, fazendo $\widetilde{q}=(x, w)$, obtemos a energia cinética nas novas coordenadas, $\widetilde{T}=\frac{1}{2}\langle\widetilde{B}(\widetilde{q}) \dot{\widetilde{q}} \mid \dot{\widetilde{q}}\rangle$, 
onde

$$
\widetilde{B}=\left[\begin{array}{cc}
\mathrm{F}+2 \mathrm{G}+\mathrm{H} \gamma^{\prime 2} & \mathrm{G}+\mathrm{H} \gamma^{\prime} \\
\mathrm{G}+\mathrm{H} \gamma^{\prime} & \mathrm{H}
\end{array}\right] .
$$

Observando que $\widetilde{B}(0,0)=I$ e usando as estimativas (3.4) tem-se

Fato 3.3 A matriz $\widetilde{B}$ pode ser escrita como $\widetilde{B}=I+\widetilde{h}$, onde $\|h\|$ é o $\left(\|\widetilde{q}\|^{\delta_{1}}\right)$ e $\left\|h^{\prime}\right\|$ é $o\left(\|\widetilde{q}\|^{\delta_{2}}\right), \operatorname{com} \delta_{1}=\min \left\{\beta_{1}-1,1\right\} e$ $\delta_{2}=\min \left\{\beta_{1}-2,0\right\}=\delta_{1}-1$.

Além disso $\widetilde{B}$ é invertível e temos $\widetilde{B}^{-1}=I+d$, onde $\|d\|$ é $o\left(\|\widetilde{q}\|^{\delta_{1}}\right)$ e $\left\|d^{\prime}\right\|$ é $o\left(\|\widetilde{q}\|^{\delta_{2}}\right)$.

Com estes ingredientes, podemos escrever as equações de Lagrange nas coordenadas $(x, w, \dot{x}, \dot{w})$.

Fato 3.4 Reduzindo à forma normal as equações de Lagrange do sistema considerado, nas coordenadas $(x, w, \dot{x}, \dot{w})$, obtemos

$$
\ddot{\widetilde{q}}=\widetilde{B}^{-1}\left(-\nabla \widetilde{\pi}+O\left(\|\widetilde{q}\|^{\delta_{2}}\|\dot{\widetilde{q}}\|^{2}\right) .\right.
$$

Daqui resulta em particular a equação,

$$
\ddot{w}=-f(x, w) \frac{\partial \widetilde{\pi}}{\partial w}-g(x, w) \frac{\partial \widetilde{\pi}}{\partial x}+O\left(\|\widetilde{q}\|^{\delta_{2}}\|\dot{\widetilde{q}}\|^{2}\right),
$$

onde $f(0,0)=1$ e $g(0,0)=0$.

Para construir o cone $C$ e a função auxiliar que nos permitirão demonstrar a existência da trajetória assintótica para a origem, será útil estudar em detalhes o comportamento de $P_{2}$ nas curvas de equação $w=a x^{c}, x>0$, para convenientes valores de $a \in \mathbf{R}$ e $c \geq 1$.

Suponhamos $P_{2} \not \equiv 0$, e tomemos $a \neq 0$ e $c \geq 1$. Na curva $w=a x^{c}, x>0$ o monômio $a_{s r} x^{s} w^{r+2}$ tem nessa curva ordem $s+c(r+2)$. 
Como para todo $(s ; r) \in \widetilde{I}$ temos $s+r+2 \leq \bar{\beta}$ podemos escolher $\bar{c} \geq 1$ tal que $\min \{s+\bar{c}(r+2):(s ; r) \in \widetilde{I}\}=\bar{\beta}$.

É claro que há apenas um $\bar{c}$ nessas condições e chamaremos $J$ ao conjunto $\{(s ; r): s+\bar{c}(r+2)=\bar{\beta}\}$. Portanto, $J \neq \emptyset \mathrm{e}$, na curva $w=a x^{c}, x>0$, tem-se

$$
P_{2}(x ; w)=P_{2}\left(x ; a x^{\bar{c}}\right)=\sum_{(s ; r) \in J} a_{s r} a^{r+2} x^{\bar{\beta}}+o\left(x^{\bar{\beta}}\right) .
$$

Consideremos o polinômio $\widetilde{P}(a)=\sum_{(s ; r) \in J} a_{s r} a^{r+2}$, vem de (3.9) que $P\left(x ; a x^{\bar{c}}\right)=\widetilde{P}(a) x^{\bar{\beta}}+o\left(x^{\beta}\right)$.

Tomemos agora $r_{0}=\min \{r \in \mathrm{N}: \exists s \in \mathrm{Q},(s ; r) \in J\}$ e observemos que existe um único $s_{0}$ tal que $\left(s_{0} ; r_{0}\right) \in J$.

Nessas condições vale que

Fato 3.50 polinômio $\widetilde{P}$ satisfaz $\widetilde{P}(0)=0$ e $\widetilde{P}(a) \geq 0$ para todo $a \in \mathrm{R}$.

Prova: Como $r_{0} \in \mathrm{N}$ resulta que $r_{0}+2 \geq 2$, logo segue da forma como foi escolhido $r_{0}$ que $\widetilde{P}$ tem uma raiz de multiplicidade maior ou igual a 2 em $a=0$.

Além disso, observemos que $P\left(x ; \bar{a} x^{\bar{c}}\right)-P(x ; 0)=\widetilde{\widetilde{P}}(\bar{a}) x^{\bar{\beta}}+$ $o\left(x^{\bar{\beta}}\right), \log$ se, por absurdo, existisse $\bar{a} \in \mathrm{R}$ tal que $\widetilde{P}(\bar{a})<0$ então $\bar{a} \neq 0$ e teríamos

$$
\lim _{x \downarrow 0} \frac{P\left(x ; \bar{a} x^{\bar{c}}\right)-P(x ; 0)}{x^{\bar{\beta}}}=\widetilde{P}(\bar{a})<0 .
$$

Dessa forma, para $x>0$ suficientemente pequeno, teríamos a desigualdade $P\left(x ; \bar{a} x^{\bar{c}}\right)<P(x ; 0)$ contrariando o fato de $\gamma$ ser a curva de mínimos vertical de $P$ e estabelecendo, por contradição, a tese. 
Lembremos agora a definição de $r_{0}$ e façamos

$$
G(a)=\sum_{\substack{(s ; r) \in J \\ r>r_{0}}} a_{s r} a^{r-\left(r_{0}+1\right)}
$$

obtendo (veja que $\left.r-\left(r_{0}+1\right)=r+2-\left(r_{0}+3\right)\right)$ :

$$
\widetilde{P}(a)=a_{s_{0} r_{0}} a^{r_{0}+2}+a^{r_{0}+3} G(a) .
$$

No futuro será útil lembrar a desigualdade

$$
\left|a^{r_{0}+3} G(a)\right| \leq\left|a^{r_{0}+3}\right| \sum_{\substack{(s, r) \in J \\ r>r_{0}}}\left|a_{s r} a^{r-\left(r_{0}+1\right)}\right|
$$

Consideremos agora, para cada número real $a>0$, o cone

$$
C_{a}=\left\{(x ; w): x>0,-a x^{\bar{c}} \leq w \leq a x^{\bar{c}}\right\} .
$$

Vamos estimar o comportamento de $P_{2}$ e de $x \frac{\partial P_{2}}{\partial x}$ em $C_{a}$.

Para isso consideremos $\bar{G}(a)=\sum_{\substack{(s ; r) \in J \\ r>r_{0}}}\left|a_{s r} a^{r-\left(r_{0}+1\right)}\right|$ e observemos que valem os seguintes resultados.

Fato 3.6 Com as notações acima temos que

(i) Para $(x ; w) \in C_{a}$ temos $\left|P_{2}(x ; w)\right| \leq\left(a_{s_{0} r_{0}}+a \bar{G}(a)\right) a^{r_{0}+2} x^{\bar{\beta}}+$ $+o\left(x^{\beta}\right)$.

(ii) Se $\delta(\underline{a})=\left(a_{s_{0} r_{0}}-a \bar{G}(a)\right) a^{r_{0}+2}, a \geq 0$ então $P_{2}\left(x ; a x^{\bar{c}}\right) \geq$ $\delta(a) x^{\bar{\beta}}+o\left(x^{\bar{\beta}}\right)$.

Prova: Para provar (i) veja que, se $(x ; w) \in C_{a}$, então $(x ; w)=$ $\left(x ; b x^{\bar{c}}\right)$, onde $-a \leq b \leq a$ e, observando que $\bar{G}$ é crescente, temos portanto

$$
\begin{aligned}
\left|P_{2}(x ; w)\right| & =a_{s_{0} r_{0}} b^{r_{0}+2} x^{\bar{\beta}}+\left|b^{r_{0}+3} G(b)\right| x^{\bar{\beta}}+o\left(x^{\bar{\beta}}\right) \leq \\
& \leq\left(a_{s_{0} r_{0}}+a \bar{G}(a)\right) a^{r_{0}+2} x^{\bar{\beta}}+o\left(x^{\beta}\right) .
\end{aligned}
$$


Quanto à segunda desigualdade temos que, por (3.9) e (3.10), $P_{2}\left(x ; a x^{\bar{c}}\right)=\widetilde{P}(a) x^{\bar{\beta}}+o\left(x^{\bar{\beta}}\right)=\left(a_{s_{0} r_{0}} a^{r_{0}+2}+a^{r_{0}+3} G(a)\right) x^{\bar{\beta}}+o\left(x^{\bar{\beta}}\right)$, $\operatorname{logo}$, como $a>0$, resulta imediatamente da definição de $\bar{G}$ e de (3.11), que (ii) vale.

Fato 3.7 Existem $a_{0}>0$ e $\varepsilon>0$ tal que, se $0<a<a_{0} e$ $(x ; w) \in C_{a}$, com $x<\varepsilon$ temos

$$
\left|x \frac{\partial P_{2}}{\partial x}(x ; w)\right|<\left(k-\frac{3}{2}\right) \delta(a) x^{\bar{\beta}}+o\left(x^{\bar{\beta}}\right) .
$$

Prova: Comecemos por notar que $x \frac{\partial P_{2}}{\partial x}(x ; w)=w^{2} \sum_{(s ; r) \in \tilde{I}} s a_{s r} x^{s} w^{r}$, logo, em $C_{a}$ temos, tomando como antes $(x ; w)=\left(x ; b x^{\bar{c}}\right)$,

$$
\begin{aligned}
\left|x \frac{\partial P_{2}}{\partial x}(x ; w)\right| & =\left|b^{r_{0}+2}\right|\left|\left(s_{0} a_{s_{0} r_{0}}+\sum_{\substack{(s ; r) \in J \\
r>r_{0}}} s a_{s r} b^{r-r_{0}}\right)\right| x^{\bar{\beta}}+o\left(x^{\bar{\beta}}\right) \leq \\
& \leq\left|\left(a_{s_{0} r_{0}}+\sum_{\substack{(s, r) \in J \\
r>r_{0}}} \frac{s}{s_{0}} a_{s r} a^{r-r_{0}}\right)\right| s_{0} a^{r_{0}+2} x^{\bar{\beta}}+o\left(x^{\bar{\beta}}\right)
\end{aligned}
$$

Da forma como foram definidos $J$ e o par $\left(s_{0} ; r_{0}\right)$ temos que se $(s ; r)$ está em $J \backslash\left\{\left(s_{0} ; r_{0}\right)\right\}$ então $s<s_{0} \leq k-2$, portanto, resulta de (3.12) que para $(x ; w) \in C_{a}$,

$$
\begin{aligned}
\left|x \frac{\partial P_{2}}{\partial x}(x ; w)\right| & \leq(k-2)\left(a_{s_{0} r_{0}}+a \bar{G}(a)\right) a^{r_{0}+2} x^{\bar{\beta}}+o\left(x^{\bar{\beta}}\right)= \\
& =(k-2)\left(\delta(a)+2 a^{r_{0}+3} \bar{G}(a)\right) x^{\bar{\beta}}+o\left(x^{\bar{\beta}}\right) .(3
\end{aligned}
$$

Observe agora que $\delta(a)$ é $O\left(a^{r_{0}+2}\right)$ e $a^{r_{0}+3} \bar{G}(a)$ é $o\left(a^{r_{0}+2}\right)$, então dado $\varepsilon>0$ existe $a_{0}=a_{0}(\varepsilon)>0$ tal que, se $0 \leq a<a_{0}$, tem-se $2 a^{r_{0}+3} \bar{G}(a) \leq \varepsilon \delta(a)$. 
Escolha agora $\varepsilon>0$ tal que $(k-2)(\varepsilon+1)<k-\frac{3}{2}$, e veja que se $a<a_{0}$, segue de (3.13), que em $C_{a}$, $\left|x \frac{\partial P_{2}}{\partial x}(x ; w)\right| \leq[(k-2)(\varepsilon+1) \delta(a)] x^{\bar{\beta}}+o\left(x^{\bar{\beta}}\right)<\left(k-\frac{3}{2}\right) \delta(a) x^{\bar{\beta}}+o\left(x^{\bar{\beta}}\right)$, que é exatamente a desigualdade desejada.

Podemos agora, enfim, demonstrar o resultado fundamental desta seção.

Lema 3.1 Existem $0<\delta<\frac{1}{4}$ e $\sigma>0$ tais que, na componente conexa $C_{1}$ de

$$
\Omega_{1}=\left\{(x ; w) \in \mathbf{R}^{2}: 0<x<\sigma, P_{2}(x ; w)<\delta x^{\bar{\beta}}\right\}
$$

que contém o segmento $(x ; 0), 0<x<\sigma$, vale

$$
x \frac{\partial P_{2}}{\partial x}(x ; w)<(k-1) \delta x^{\bar{\beta}} .
$$

Prova: Manteremos nesta demonstração as notações que introduzimos anteriormente nesta seção.

Observemos que $\lim _{a \downarrow 0} \frac{\delta(a)}{a^{r_{0}+2}}=a_{s_{0} r_{0}}>0$, assim, diminuindo eventualmente o $a_{0}$ tomado acima, temos $\delta(a)>0$, para $0<a \leq a_{0}$.

Note também que, se $a>0, \frac{\delta(a)}{a}=\left(a_{s_{0} r_{0}}-a \bar{G}(a)\right) a^{r_{0}+1}$, portanto, como $r_{0}+1 \geq 1$ podemos escolher $a_{0}$ suficientemente pequeno para que a função $\delta$ seja crescente em $\left[0 ; a_{0}\right]$.

Agora, do fato 3.6 resulta $P_{2}\left(x ; \pm a_{0} x^{\bar{c}}\right) \geq \delta\left(a_{0}\right) x^{\beta}+o\left(x^{\bar{\beta}}\right)$, fazendo então $\delta=\frac{\delta\left(a_{0}\right)\left(k-\frac{3}{2}\right)}{k-\frac{4}{3}}$ tem-se que existe $\sigma>0$ tal que, se $0<x \leq \sigma$,

$$
P_{2}\left(x ; \pm a_{0} x^{\bar{c}}\right) \geq \delta x^{\bar{\beta}} .
$$

Isto mostra que se $\bar{C}$ é a componente conexa de

$$
\Omega_{1}=\left\{(x ; w) \in \mathrm{R}^{2}: 0<x \leq \sigma, P_{2}(x ; w)<\delta x^{\bar{\beta}}\right\}
$$


que contém o segmento $(x ; 0), 0<x<\sigma$, então $\bar{C} \subset C_{a_{0}}$.

Por outro lado, como em $\left[0 ; a_{0}\right], \delta(a)$ é crescente, é claro a partir do fato 3.7 que, eventualmente reduzindo um pouco o valor de $\sigma$ temos, para $(x ; w) \in C_{a_{0}}$,

$$
\left|x \frac{\partial P_{2}}{\partial x}(x ; w)\right| \leq \frac{k-1}{k-\frac{4}{3}}\left(k-\frac{3}{2}\right) \delta\left(a_{0}\right) x^{\bar{\beta}}=\delta(k-1) x^{\bar{\beta}} .
$$

Como $\bar{C} \subset C_{a_{0}},(3.15)$ encerra a demonstração.

\subsection{O Teorema de Instabilidade}

Nesta seção mostraremos que, nas condições em que estamos trabalhando, existe uma trajetória assintótica para a origem nas equações (1.1).

Isso será feito construindo no espaço de fase $(x, w, \dot{x}, \dot{w})$ um cone $C$ e uma função auxiliar $V$ que, como nas seções (2) e (3) do Capítulo 2, será usada para estabelecer que existe em $C$ uma trajetória assintótica.

Uma vez que a função $\varphi$ que usamos para determinar as novas variáveis $(x ; w)$ foi definida em $\mathcal{F}$, que não é uma vizinhança aberta da origem, convém relembrar aqui a observação feita na seção anterior. Definimos $\varphi$ na faixa $\mathcal{F}=\left\{(x ; y) \in \mathrm{R}^{2}: 0<x<\varrho\right\}$ e notamos que sua extensão a $\overline{\mathcal{F}}$ é um difeomorfismo.

Notemos que, como $\varphi(0 ; 0)=(0 ; 0)$, se provarmos que há uma solução $\psi(t)=(x(t) ; w(t))$ das equações $\left(^{*}\right)$ definida em $(-\infty ; 0]$, com $\psi(t) \in \mathcal{F}$ tal que $\lim _{t \rightarrow-\infty} \psi(t)=(0 ; 0)$, então verifica-se que a função $\bar{\psi}(t)=\varphi^{-1}(\psi(t))$, também definida em $(-\infty ; 0]$, é uma solução de (1.1) e

$$
\lim _{t \rightarrow-\infty} \bar{\psi}(t)=(0 ; 0)=\lim _{t \rightarrow-\infty} \varphi^{-1}(\psi(t))=(0 ; 0) .
$$


Portanto, $\bar{\psi}$ será uma trajetória assintótica no passado à origem das equações (1.1).

Vamos agora definir a função auxiliar $V$ já citada.

Seja $V: \mathcal{F} \times \mathrm{R}^{2} \longrightarrow \mathrm{R}$ dada por

$$
V(x ; w ; \dot{x} ; \dot{w})=\frac{\dot{w}^{2}}{2 f(x ; w)}+P_{2}(x ; w) .
$$

Um cálculo direto fornece, graças a (3.8),

$$
\begin{aligned}
\dot{V} & =\left(P_{2}\right)_{x} \dot{x}+\left(P_{2}\right)_{w} \dot{w}+\frac{1}{f} \dot{w} \ddot{w}-\frac{1}{f^{2}} \dot{f} \dot{w}^{2} \\
& =\left(P_{2}\right)_{x} \dot{x}+\left(P_{2}\right)_{w} \dot{w}-\frac{1}{f^{2}} \dot{f} \dot{w}^{2}+ \\
& +\frac{1}{f}\left[-f \widetilde{\pi}_{w}-g \widetilde{\pi}_{x}+o\left(\|(x ; w)\|^{\delta_{2}}\|(\dot{x} ; \dot{w})\|^{2}\right)\right] \dot{w}
\end{aligned}
$$

onde $\dot{f}=\left(f_{x}\right) \dot{x}+\left(f_{w}\right) \dot{w}=O\left(\|(x ; w)\|^{\delta_{2}}\|(\dot{x} ; \dot{w})\|\right)$.

Logo, se considerarmos $\widetilde{V}=\frac{V}{x^{\bar{\beta}}}$, vem que $\dot{\widetilde{V}}=\frac{\dot{V}}{x^{\bar{\beta}}}-\frac{\bar{\beta} \dot{x} V}{x^{\bar{\beta}+1}}$. Portanto, usando (3.17), obtemos

$$
x^{\bar{\beta}+1} \dot{\widetilde{V}}=x \dot{V}-\bar{\beta} V \dot{x}=\left(x\left(P_{2}\right)_{x}-\bar{\beta} V\right) \dot{x}+R(x ; w ; \dot{x} ; \dot{w}),
$$

com

$$
\begin{gathered}
R(x ; w ; \dot{x} ; \dot{w})= \\
-\frac{1}{f^{2}} \dot{f} \dot{w}^{2}+\frac{x \dot{w}}{f}\left[-f\left(\widetilde{\pi}_{w}-\left(P_{2}\right)_{w}\right)-g \widetilde{\pi}_{x}+o\left(\|(x ; w)\|^{\delta_{2}}\|(\dot{x} ; \dot{w})\|^{2}\right)\right] .
\end{gathered}
$$

Estes cálculos serão fundamentais. Agora definiremos o cone $C$ em que trabalharemos por:

$C=\left\{(x ; w ; \dot{x} ; \dot{w}) \in \mathcal{F} \times \mathrm{R}^{2}: \begin{array}{c}E(x ; w ; \dot{x} ; \dot{w})=0,0<x<\sigma_{1}<\sigma \\ (x ; w) \in C_{1}, V(x ; w ; \dot{x} ; \dot{w})<\delta x^{\bar{\beta}}\end{array}\right\}$ 
onde $\delta, \sigma$ e $C_{1}$ estão dados no lema $3.1, E=T+\tilde{\pi}$ é a energia associada ao lagrangeano e $\sigma_{1}$ é um número suficientemente pequeno que será determinado em breve.

Mostraremos agora uma desigualdade importante, expressa no seguinte resultado:

Lema 3.2 Existe $\varepsilon_{0}>0$ tal que, se $0<\varepsilon<\varepsilon_{0}$ há $\varrho>0$ tal que se $\|(x ; w)\| \leq \varrho e(x ; w ; \dot{x} ; \dot{w}) \in C$ então

$$
\frac{1}{(1+\varepsilon)} x^{\beta} \leq \dot{x}^{2} \leq \frac{4}{1-\varepsilon} x^{\beta}
$$

Prova: Seja $\varepsilon>0$. Como $B(0)=I d$, numa vizinhança da origem temos

$$
(1-\varepsilon) \frac{\dot{x}^{2}+\dot{w}^{2}}{2} \leq T(x ; w ; \dot{x} ; \dot{w}) \leq(1+\varepsilon) \frac{\dot{x}^{2}+\dot{w}^{2}}{2} .
$$

Em $C$ a energia é nula, portanto $(1+\varepsilon) \frac{\dot{x}^{2}+\dot{w}^{2}}{2}+\tilde{\pi}(x ; w)>0$, e, usando agora o Fato 3.4 , isto mostra que, para $(x ; w ; \dot{x} ; \dot{w}) \in C$

$$
(1+\varepsilon) \frac{\dot{x}^{2}}{2}>-\left[-x^{\beta}+P_{2}(x ; w)+o\left(\|(x ; w)\|^{\beta}\right)+(1+\varepsilon) \frac{\dot{w}^{2}}{2}\right] .
$$

Analisando agora $V$ vemos que, em $C$,

$$
V(x ; w ; \dot{x} ; \dot{w})=\frac{\dot{w}^{2}}{2 f}+P_{2}(x ; w)<\delta x^{\beta}
$$

portanto, usando as estimativas do Fato (3.3) para $f$, temos que $f(x, w)=1+o\left(\widetilde{q}^{\delta_{1}}\right)=1+o(1)$ e assim, se $0<x<\tilde{\sigma}$, a desigualdade $\frac{\dot{w}^{2}}{2}<f\left(\delta x^{\beta}-P_{2}(x ; w)\right)<\left(1+\varepsilon_{1}\right)\left(\delta x^{\beta}-P_{2}(x ; w)\right)$, onde $\varepsilon_{1}>0$ é escolhido de forma a termos $\varepsilon+\varepsilon_{1}+\varepsilon \varepsilon_{1}<2 \varepsilon$.

Multiplicando a última desigualdade por $(1+\varepsilon)$ e substituindo em (3.22), obtemos

$$
\frac{1+\varepsilon}{2} \dot{x}^{2}>x^{\beta}-P_{2}(x ; w)+(1+\varepsilon)\left(1+\varepsilon_{1}\right)\left(P_{2}(x ; w)-\delta x^{\beta}\right)+\text { tos }=
$$




$$
\begin{gathered}
=x^{\beta}-\delta(1+\varepsilon)\left(1+\varepsilon_{1}\right) x^{\beta}+\left(\varepsilon+\varepsilon_{1}+\varepsilon \varepsilon_{1}\right) P_{2}(x ; w)+\text { tos }= \\
=(1-\delta) x^{\beta}-\left(\delta x^{\beta}-P_{2}(x ; w)\right)\left(\varepsilon+\varepsilon_{1}+\varepsilon \varepsilon_{1}\right)+\text { tos. }
\end{gathered}
$$

onde tos são termos em $o\left(\|(x ; w)\|^{\beta}\right)$.

Como em $C$ temos $\delta x^{\beta}>P_{2}(x ; w)$, se lembrarmos a forma como escolhemos $\varepsilon_{1}$ e usarmos que $1-\delta>\frac{3}{4}$ e (3.23), concluímos que

$$
\frac{1+\varepsilon}{2} \dot{x}^{2}>\frac{3}{4} x^{\beta}+2 \varepsilon\left(P_{2}(x ; w)-\delta x^{\beta}\right)+o\left(\|(x ; w)\|^{\beta}\right) .
$$

Notemos agora que existe $\sigma>0$ tal que $P_{2}(x ; w)>0$ se $0<$ $x<\sigma$, logo resulta de (3.24) que

$$
\frac{1+\varepsilon}{2} \dot{x}^{2}>\left(\frac{3}{4}-2 \delta \varepsilon\right) x^{\beta}+o\left(\|(x ; w)\|^{\beta}\right) .
$$

Então, se escolhermos $\varepsilon_{0}>0$ tal que $\frac{3}{4}-2 \delta \varepsilon_{0}>\frac{1}{2}$, tem-se que, para $\varepsilon \leq \varepsilon_{0}$, podemos escolher $\varrho_{1}>0\left(\operatorname{com} \varrho_{1} \leq \sigma\right)$ tal que, para os pontos de $C$ tais que $\|(x ; w)\| \leq \varrho_{1}$, a desigualdade da esquerda em (3.20) fica satisfeita.

Quanto à outra desigualdade, notamos que segue de (3.21) que, em $C$, temos $\frac{1-\varepsilon}{2} \dot{x}^{2}+\frac{1-\varepsilon}{2} \dot{w}^{2}+\widetilde{\pi}(x ; w)<0$, portanto

$$
\frac{1-\varepsilon}{2} \dot{x}^{2}<-\left[\widetilde{\pi}(x ; w)+\frac{1-\varepsilon}{2} \dot{w}^{2}\right] \leq-\widetilde{\pi}(x ; w) .
$$

Como $P_{2}(x ; w)>0$ resulta do Fato 3.4 que, em $C, \tilde{\pi}(x ; w)>$ $-x^{\beta}+o\left(\|(x ; w)\|^{\beta}\right)$.

Portanto, existe $\varrho_{2}>0$ tal que, se $\|(x ; w)\| \leq \varrho_{2}$ e $(x ; w ; \dot{x} ; \dot{w}) \in$ $C$, temos $-\tilde{\pi}(x ; w) \leq 2 x^{\beta}$. Isto, unido a (3.25), estabelece imediatamente a desigualdade da direita em (3.20).

Agora, basta considerar $\varrho=\min \left\{\varrho_{1}, \varrho_{2}\right\}$ e tem-se a tese. 
Lema 3.3 Para todo real $\lambda>0$, existe $\sigma_{1}, 0<\sigma_{1}<\sigma$ tal que em $\partial C \backslash(0 ; 0) \cap\{(x ; w):|w| \leq \lambda x\}$ a função $\dot{\vec{V}}$ não se anula.

Prova: Basta mostrar que $x^{\bar{\beta}+1} \dot{\widetilde{V}}$ não se anula nesse conjunto, pois $x>0$ em $\partial C \backslash(0 ; 0) \cap\{(x ; w):|w| \leq \lambda x\}$.

Pela fórmula (3.18) $x^{\bar{\beta}+1} \dot{\widetilde{V}}=x \dot{V}-\bar{\beta} \dot{x} V$.

Analisemos a parcela $x \dot{V}$; sabemos, por (3.17), que

$$
x \dot{V}=x\left[\left(P_{2}\right)_{x} \dot{x}+\left(P_{2}\right)_{w} \dot{w}-\frac{1}{f^{2}} \dot{f} \dot{w}^{2}-\widetilde{\pi}_{w} \dot{w}+R(x ; w ; \dot{x} ; \dot{w})\right],
$$

onde $R(x ; w ; \dot{x} ; \dot{w})=\frac{1}{f}\left[-g \widetilde{\pi}_{x}+o\left(\|(x ; w)\|^{\delta_{2}}\|(\dot{x} ; \dot{w})\|^{2}\right)\right] \dot{w}$.

Notemos agora que:

(i) Como a energia em $C$ é nula, segue-se do lema 2 e da definição de $C_{1}$ que $\dot{w}$ é $O\left(x^{\frac{\bar{\beta}}{2}}\right)$ na fronteira de $C$;

(ii) Do Fato (3.3) temos que $\delta_{2}>-1$, e disso resulta que a parcela $\frac{x}{f} o\left(\|(x ; w)\|^{\delta_{2}}\|(\dot{x} ; \dot{w})\|^{2}\right)$ é $o\left(x^{\bar{\beta}}\right)$ em $\partial C$;

(iii) Uma vez que $g(0 ; 0)=0$, vem de $P=j^{k} \widetilde{\pi}$ e de (3.13) que, em $\partial C, x g \widetilde{\pi}_{x}$ é $o\left(x^{\bar{\beta}}\right)$.

(iv) De (ii) e (iii) é imediato que, na fronteira de $C, x R=\dot{w} R_{1}$, onde $R_{1}$ é $o\left(x^{\bar{\beta}}\right)$, logo, segue-se de (i) que $x R$ é $o\left(x^{\frac{3 \bar{\beta}}{2}}\right)$ em $\partial C$

(v) Como $\dot{f}=\left(f_{x}\right) \dot{x}+\left(f_{w}\right) \dot{w}$, resulta do Fato 3.3, do Lema 3.2, e de (i) que $\dot{f} \dot{w}^{2}$ é $O\left(x^{\frac{3 \bar{B}}{2}+\delta_{2}}\right)$ em $\partial C$, assim a parcela $\frac{x}{f^{2}} \dot{f} \dot{w}^{2}$ é $o\left(x^{\frac{3 \bar{\beta}}{2}}\right)$, pois $\delta_{2}>-1$,

Resta, portanto, analisar $x\left[\left(P_{2}\right)_{x} \dot{x}+\left(P_{2}\right)_{w} \dot{w}-\tilde{\pi}_{w} \dot{w}\right]-\bar{\beta} \dot{x} V$. 
Usemos, mais uma vez, que $P=j^{k} \widetilde{\pi}$ e (3.13), para ver que na fronteira de $C$ temos $\widetilde{\pi}_{w}=\left(P_{2}\right)_{w}+o\left(x^{\bar{\beta}-1}\right)$ e, portanto, graças a (i),

$$
x\left[\left(P_{2}\right)_{x} \dot{x}+\left(P_{2}\right)_{w} \dot{w}-\widetilde{\pi}_{w} \dot{w}\right]-\bar{\beta} \dot{x} V=\left(x\left(P_{2}\right)_{x}-\bar{\beta} V\right) \dot{x}+o\left(x^{\frac{3 \bar{\beta}}{2}}\right) .
$$

Notemos agora que:

(vi) Em $\partial C$ temos $V=\delta x^{\bar{\beta}}$ e $(x ; w) \in \bar{\Omega}_{1}$, logo segue-se do lema 1 que, $x\left(P_{2}\right)_{x}<(k-1) \delta x^{\bar{\beta}}$

(vii) Como $\bar{\beta}>k-1$ resulta que, em $\partial C$, temos $x\left(P_{2}\right)_{x}-\bar{\beta} V<0$ e $\left|x\left(P_{2}\right)_{x}-\bar{\beta} V\right| \geq(\bar{\beta}-(k-1)) \delta x^{\bar{\beta}}$.

Portanto, resulta do Lema (3.2) e de (vii) que se $(x ; w ; \dot{x} ; \dot{w}) \in$ $\partial C \backslash(0 ; 0)$ temos

$$
\left|\left(x\left(P_{2}\right)_{x}-\bar{\beta} V\right) \dot{x}\right| \geq(\bar{\beta}-(k-1)) \delta x^{\bar{\beta}}|\dot{x}| \geq \frac{(\bar{\beta}-(k-1)) \delta}{(1+\varepsilon)} x^{\frac{3 \bar{\beta}}{2}} .
$$

É claro que de (iv), (v), (3.26) e (3.27) segue-se a tese.

Estamos prontos para enunciar o nosso resultado principal:

Teorema 3.1 Quando $n=2$, existe uma trajetória $\phi(t)$ assintótica à origem, solução do sistema (1.1) e, para $t>t_{0}$ suficientemente grande, $\phi(t) \in C \backslash(0 ; 0) \cap\{(x ; w):|w| \leq 2 x\}$.

Prova: Para facilitar a notação, chamaremos de $\Omega_{1}$ ao conjunto

$$
\Omega_{1}=C \backslash(0 ; 0) \cap\{(x ; w ; \dot{x} ; \dot{w}):|w| \leq 2 x \text { e } \dot{x}<0\} .
$$

Primeiro notamos que no Lema 3.2 , se escolhermos $\sigma_{1}$ suficientemente pequeno, teremos que, para $0<x<\sigma_{1}, \dot{x}<-\frac{1}{2} x^{\frac{\bar{\beta}}{2}}$. 
Por outro lado, da própria definição de $C$, sabemos que se $|x|$ for suficientemente pequeno, e se $(x ; w) \in \Omega_{1}$, então

$$
|\dot{w}|<\frac{4}{3} \delta x^{\frac{\bar{\beta}}{2}}<2|\dot{x}| .
$$

Das duas últimas afirmações segue facilmente que, para qualquer solução $\bar{\phi}(t)=(\bar{x}(t) ; \bar{w}(t))$ de (3.4), se num instante $t_{1}$ vale que $\bar{\phi}\left(t_{1}\right) \in \bar{\Omega}_{1}$ e $\left|\bar{w}\left(t_{1}\right)\right|=2 \bar{x}\left(t_{1}\right)$, então para qualquer $t$ num intervalo $\left[t_{1}, t_{1}+\varepsilon\right)$ com $\varepsilon$ pequeno, temos que $\bar{\phi}(t) \notin \bar{\Omega}_{1}$.

Este fato, aliado ao Lema 3.3, mostra-nos que toda solução que atinja $\bar{\Omega}_{1} \backslash(0 ; 0)$ sairá imediatamente deste conjunto.

Finalmente, relembramos que $\dot{x}<0$ em $\Omega_{1}$. Logo, podemos encerrar a demonstração de maneira inteiramente análoga ao Teorema 2.2 do Capítulo 2. Apesar de não nos encontrarmos na hipótese do Teorema citado, visto que $\Omega_{1}$ não é aberto (pois fizemos uso do nível de energia 0), a demonstração é, mutatis mutantis, a mesma.

Como no teorema citado, chegamos ao resultado enunciado: a existência de uma trajetória assintótica.

Finalmente, para terminarmos este capítulo, apresentamos dois exemplos de energias potenciais que satisfazem as hipóteses do último teorema, e que não satisfaziam nenhum dos critérios anteriores de instabilidade.

\section{Exemplo 3.1 Seja}

$$
\pi_{1}(x, y)=x^{2} y^{2}-x^{5}+R(x, y),
$$

onde $R$ é de classe $\mathcal{C}^{2}$ e $R=o\left(\|(x ; y)\|^{5}\right)$. Então, qualquer que seja a energia cinética $T$ de classe $\mathcal{C}^{2}$, existe uma trajetória assintótica à $(0 ; 0)$, solução das equações do movimento para o lagrangeano $T-\pi_{1}$. 
Prova: Temos que $j^{5} \pi_{1}(x, y)=x^{2} y^{2}-x^{5}$ e, portanto, $j^{5} \pi_{1}(x, 0)=$ $-x^{5}$, o que mostra que $\pi_{1}$ não tem mínimo (ver Teorema 5.1). As outras hipóteses do Teorema 3.1 são facilmente verificadas.

Vale notar que, apesar do $j^{4} \pi_{1}$ ter mínimo brando na origem, não existe uma mudança de variáveis do tipo "Spliting lemma" que transformem o jato 4 desta energia em $-u^{4}$. Desta forma, uma técnica como a desenvolvida em [MMN] não poderia ser utilizada.

Exemplo 3.2 Seja

$$
\pi_{2}(x, y)=y^{4}-4 y x^{6}+S(x, y),
$$

onde $S$ é de classe $\mathcal{C}^{2}$ e $S=o\left(\|(x ; y)\|^{8}\right)$. Então, qualquer que seja a energia cinética $T$ de classe $\mathcal{C}^{2}$, existe uma trajetória assintótica à $(0 ; 0)$, solução das equações do movimento para o lagrangeano $T-\pi_{2}$.

Prova: Outra vez, temos que $j^{8} \pi_{2}\left(x, x^{2}\right)=-3 x^{8}$, o que mostra que $\pi_{2}$ não tem mínimo (ver outra vez o Teorema 5.1). As outras hipóteses do Teorema 3.1 são facilmente verificadas. 


\section{Capítulo 4 \\ Influência da Energia Cinética na Estabilidade do Equilíbrio}

\subsection{Introdução}

Este capítulo visa estudar de que maneira a energia cinética pode influir na estabilidade e no comportamento local das soluções de um sistema lagrangeano, mesmo quando a energia potencial é um polinômio. A principal conjectura neste sentido, que nos foi apresentada por nosso orientador, é:

Conjectura 4.1 Seja $\Omega$ um aberto do $\mathbf{R}^{n}, T_{1}$ e $T_{2} \in \mathcal{C}^{2}(\Omega \times$ $\left.\mathbf{R}^{n}, \mathbf{R}\right)$ duas energias cinéticas positivas definidas nas velocidades e seja $\pi$ uma energia potencial, também de classe $\mathcal{C}^{2}$, com um ponto crítico em 0 . Se $(0,0)$ é um ponto de equilíbrio instável segundo Liapounof para as equações do movimento do lagrangeano $L_{1}=T_{1}-\pi$, então $(0,0)$ também é um equilíbrio instável para as equações do movimento do lagrangeano $L_{2}=T_{2}-\pi$.

Esta conjectura já é conhecida por alguns professores do Departamento de Matemática Aplicada do IME-USP há alguns anos e, apesar do charme (na nossa opinião) e da importância que um resultado positivo nesta direção possuiria, muito pouco foi feito e o 
problema permanece virtualmente desconhecido na literatura. O único trabalho que conhecemos neste campo é o realizado por Garcia,S(Ver [Gar-S.]) que exibe um exemplo de sistema lagrangeano não conservativo onde a conjectura é falsa.

Nosso objetivo neste capítulo é apresentar um exemplo em sistemas lagrangeanos conservativos com dois graus de liberdade onde, apesar de não rejeitar a conjectura, este exemplo mostra que a influência da energia cinética não é desprezível. Apresentaremos uma energia potencial analítica $\pi$, com um ponto de sela na origem, e duas energias potenciais (também analíticas) $T_{1}$ e $T_{2}$, estritamente convexas em $\dot{q}$, numa vizinhança da origem. $\mathrm{O}$ equilíbrio do sistema $L_{1}=T_{1}+\pi$ terá uma variedade instável de dimensão dois (e logo, pelo princípio de reversibilidade, uma variedade estável de dimensão dois), enquanto que no sistema $L_{2}=T_{2}+\pi$ existe apenas uma trajetória assintótica à origem.

Por simplicidade, iremos trabalhar com o formalismo hamiltoniano, e suas equações

$$
\begin{aligned}
& \dot{p}_{i}=\frac{\partial H}{\partial q_{i}}, \\
& \dot{q}_{i}=-\frac{\partial H}{\partial p_{i}} .
\end{aligned}
$$

Isto claramente não invalidará os resultados para sistemas lagrangeanos, visto que as energias cinéticas são localmente estritamente convexas.

\subsection{O Exemplo}

Nossa energia potencial será

$$
\pi\left(q_{1} ; q_{2}\right)=\frac{q_{1}^{3}-q_{2}^{10}}{2}
$$


e as energias cinéticas serão

$$
\begin{aligned}
& T_{1}=\frac{\left(p_{1}^{2}+p_{2}^{2}\right)}{2}, \\
& T_{2}=\frac{\left(1+q_{1}\right)\left(p_{1}^{2}+p_{2}^{2}\right)}{2} .
\end{aligned}
$$

No primeiro caso, temos as equações

$$
\begin{aligned}
\dot{q}_{1} & =p_{1} \\
\dot{p}_{1} & =\frac{-3\left(q_{1}\right)^{2}}{2} \\
\dot{q}_{2} & =p_{2} \\
\dot{p}_{2} & =\frac{10\left(q_{2}\right)^{9}}{2} .
\end{aligned}
$$

Como as equações claramente se desacoplam em dois sistemas mecânicos unidimensionais, vemos facilmente que, para quaisquer $\left(q_{1}, q_{2}\right)$ com $q_{2} \leq 0$, existe um único $\left(p_{1}, p_{2}\right)$ tal que a solução com condições iniciais $\left(q_{1}, q_{2}, p_{1}, p_{2}\right)$ tende à origem quando $t \rightarrow \infty$.

O segundo exemplo requererá um pouco mais de trabalho. Suas equações são

$$
\begin{aligned}
\dot{q}_{1} & =\left(1+q_{1}\right) p_{1} \\
\dot{p}_{1} & =\frac{-3\left(q_{1}\right)^{2}}{2}-\frac{p_{1}^{2}+p_{2}^{2}}{2} \\
\dot{q}_{2} & =\left(1+q_{1}\right) p_{2} \\
\dot{p}_{2} & =\frac{10\left(q_{2}\right)^{9}}{2} .
\end{aligned}
$$

Vemos facilmente que existe uma trajetória assintótica a 0 , onde $p_{2}(t)=q_{2}(t)=0$. Vamos mostrar que ela é única. 
Seja então, por contradição, $\phi(t)=\left(q_{1}, q_{2}, p_{1}, p_{2}\right)(t)$ uma trajetória assintótica à origem, solução de 4.2 tal que $q_{2}(t)$ não é constantemente nula, e seja $t_{0}$ suficientemente grande de forma que $\left|q_{1}(t)\right|<\frac{1}{2}$, para todo $t>t_{0}$. Admitiremos que $t_{0}=0$.

Note que $\dot{p}_{1}(t)<0$, para todo $t \in \mathbf{R}$, e portanto, como $\lim _{t \rightarrow \infty} \phi(t)=0, p_{1}(t)>0$.

Este último fato implica que $\dot{q}_{1}>0$ para todo instante $t$ positivo, e novamente obtemos que $q_{1}(t)<0$, qualquer que seja $t>0$.

Notemos também que, para todo $t>0$, vale que

$$
\frac{d\left(p_{2} q_{2}\right)}{d t}=\dot{p}_{2} q_{2}+p_{2} \dot{q}_{2}>0
$$

e portanto $q_{2}(t) p_{2}(t)>0$, para todo $t>0$.

Lema 4.1 Para todo $t>0$, temos que $\left|q_{1}(t)\right| \geq\left|p_{1}(t)\right|^{\frac{2}{3}}$.

Prova: Consideramos a função auxiliar $V\left(q_{1}, p_{1}\right)=\frac{q_{1}^{3}+p_{1}^{2}}{2}$, e calculamos a sua derivada com respeito ao tempo

$$
\dot{V}=\frac{3 q_{1}^{2} \dot{q}_{1}}{2}+p_{1} \dot{p}_{1}=\frac{3 q_{1}^{2}\left(1+q_{1}\right) p_{1}}{2}+p_{1} \frac{-3\left(q_{1}\right)^{2}}{2}-\frac{p_{1}^{2}+p_{2}^{2}}{2}<0 .
$$

Claramente, $V$ decresce com o tempo.

Além disso, como $\lim _{t \rightarrow \infty} V\left(q_{1}(t), p_{1}(t)\right)=0$, temos que $\frac{q_{1}^{3}+p_{1}^{2}}{2}>0$, o que mostra o resultado

Lema 4.2 Para todo $t>0$, temos que $\left|p_{2}(t)\right| \geq\left|q_{2}(t)\right|^{5}$.

Prova: Considere a função auxiliar $U\left(q_{2}, p_{2}\right)=\frac{-q_{2}^{10}+p_{2}^{2}}{2}$. Calculando a sua derivada ao longo das trajetórias obtemos

$$
\dot{U}=\frac{-10 q_{2}^{9} \dot{q}_{2}}{2}+p_{2} \dot{p}_{2}=\frac{-10 q_{1} q_{2}^{9} p_{2}}{2} \leq 0
$$


onde a última desigualdade é uma consequência de $q_{1}(t)<0$ e de $q_{2}(t) p_{2}(t)<0$.

Como $\lim _{t \rightarrow \infty} U\left(q_{2}(t), p_{2}(t)\right)=0$, temos que $\frac{-q_{2}^{10}(t)+p_{2}^{2}(t)}{2}>0$, para todo instante $t$ positivo, como foi enunciado.

Teorema 4.1 Seja $\phi(t)=\left(p_{1}(t), p_{2}(t), q_{1}(t), q_{2}(t)\right)$ uma solução de (4.2) tal que $\lim _{t \rightarrow \infty}\|\phi(t)\|=0$. Então $p_{2}(t)=q_{2}(t)=0$.

Prova: Para não carregarmos na notação, admitiremos que os valores iniciais são $\left(q_{1}, p_{1}, q_{2}, p_{2}\right)(0)=\left(c_{0}, c_{1}, c_{2}, c_{3}\right)$, onde $c_{2}<$ $0<c_{3}$.

Como $p_{2}>\left|q_{2}\right|^{5}$ e $\dot{p}_{2}=10\left(q_{2}\right)^{9}$, temos que

$$
\dot{p}_{2}>-10\left(p_{2}\right)^{\frac{9}{5}} \text {. }
$$

Se lembrarmos do Lema de comparação 2.1 do Capítulo 2, temos que

$$
p_{2}(t)>\left(c_{3}^{-\frac{4}{5}}+8 t\right)^{-\frac{5}{4}}
$$

Logo existe $K_{1}$ tal que $p_{2}(t)>K_{1}\left(t^{-\frac{5}{4}}\right)$, para todo $t>1$.

Por outro lado, verifica-se que $\dot{p}_{1}<-3 \frac{q_{1}^{2}}{2}$ e, do Lema 4.1, $\left|q_{1}\right| \geq p_{1}^{\frac{2}{3}}$ de forma que

$$
p_{1}(t)<\left(c_{1}^{-\frac{1}{3}}+\frac{1}{2} t\right)^{-3} .
$$

Portanto, existe $K_{2}$ tal que, para $t>1, p_{1}(t)<K_{2}\left(t^{-3}\right)$.

Definimos agora, para $t>1$,

$$
f(t)=\int_{t}^{\infty} \frac{p_{2}^{2}(s)}{2} d s>\frac{K_{1}^{2}}{3} t^{-\frac{3}{2}} .
$$

Note que, como $\dot{p}_{1}(t)<-\frac{p_{2}^{2}(t)}{2}$, segue de (4.4) e (4.5) que, para $t>1$

$$
\lim _{s \rightarrow \infty} p_{1}(s)<p_{1}(t)-f(t)<K_{2} t^{-3}-\frac{K_{1}^{2}}{3} t^{-\frac{3}{2}} .
$$


Mas esta desigualdade claramente implica em $\lim _{t \rightarrow \infty} p_{1}(t)<0$, o que contraria a hipótese de $\phi$ ser assintótica à origem. 


\section{Apêndice K-Decidibilidade e Curva de Mínimos Verticais}

\subsection{Introdução}

No capítulo central da tese, utilizamos na demonstração uma propriedade de polinômios de duas variáveis relacionadas ao conceito de K-decidibilidade. Este apêndice se presta a demonstrar estas propriedades.

Falando livremente, uma função $f$ de n-variáveis nos reais é dita k-decidível num ponto crítico $P$, se for possível determinar o comportamento de $f$ quanto a extremo em $P$ (isto é, determinar se $P$ é um ponto de máximó local, mínimo local ou sela de $f$ ) apenas através da análise do jato de ordem $k$ de $f$ em $P$. De forma um pouco mais precisa, temos:

Definição 5.1 Uma função $f \in J^{k}(\widehat{P}, \mathbf{R})$ (Funções com jato de ordem $k$ em $P$ ) chama-se $k$ - decidivel se, para qualquer função $g \in J^{k}(P, \mathbf{R})$, com $j_{P}^{k} f(x)=j_{P}^{k} g(x)$, vale uma das seguintes afirmações:

- Se $P$ é um ponto de máximo local de $f$, então $P$ também é um ponto de máximo local de $g$. 
- Se $P$ é um ponto de mínimo local de $f$, então $P$ também é um ponto de minimo local de $\mathrm{g}$.

- Se P é um ponto de sela de $f$, então $P$ também é um ponto de sela de $g$.

Este conceito de $k$-decidibilidade foi introduzido por BaroneNetto (Veja [B]) em sua tese de livre-docência, apresentada em 1980. Uma caracterização das funções $k$-decidíveis foi dada nesta tese.

Vale notar que, $f$ é $k$ decidível em $P$ se e só se o $j_{P}^{k} f(x)$ também for k-decidível. Como $j^{k} f(P)$ é um polinômio, Barone-Netto mostrou que existe uma curva algébrica

$$
\begin{aligned}
\gamma_{-} & :[0, \varepsilon] \rightarrow \mathbf{R}^{n} \\
\left\|\gamma_{-}(a)-P\right\| & =a \\
\min _{\|x-P\|=a} j_{P}^{k} f(x) & =j_{P}^{k} f\left(\gamma_{-}(a)\right) .
\end{aligned}
$$

Esta curva $\gamma$ costuma ser chamada curva de mínimos radiais. Também existe uma curva de máximos radiais, denotada por $\gamma_{+}$.

Barone-Netto mostrou que:

Teorema $5.1 f$ é $k$-decidivel em $P$ se, e só se, existe uma constante $C>0$ tal que

$$
\begin{aligned}
& \lim _{t \rightarrow 0^{+}} \frac{\left\|j_{P}^{k}\left(\gamma_{-}(t)\right)-j_{P}^{k}(P)\right\|}{\left\|\gamma_{-}(t)-P\right\|^{k}}>C, \\
& \lim _{t \rightarrow 0^{+}} \frac{\left\|j_{P}^{k}\left(\gamma_{+}(t)\right)-j_{P}^{k}(P)\right\|}{\left\|\gamma_{+}(t)-P\right\|^{k}}>C .
\end{aligned}
$$

Outros trabalhos relacionados a $k$-decidibilidade foram realizados. O Leitor interessado poderá consultar [BGZ]. 


\subsection{Curva de Mínimos Vertical}

Nesta seção mostraremos que existe a curva de mínimos vertical com as propriedades descritas no Capítulo 3 .

Seja $f: \Omega \longrightarrow \mathrm{R}$ uma função definida na vizinhança aberta $\Omega$ da origem de $\mathrm{R}^{2}$, com $f(O)=0$, que tem jato punctual de ordem $k$ na origem e tal que:

i $j_{0}^{k} f$ mostra que $f$ não tem mínimo na origem.

ii $j_{0}^{k-1} f$ não mostra que $f$ não tem mínimo na origem.

Para não sobrecarregarmos a notação, faremos nesta seção $Q=$ $j_{0}^{k} f$.

Lembramos que, como uma consequência direta do Teorema (5.1), podemos provar que $Q$ mostra que $f$ não tem mínimo na origem se, e só se, vale que

$$
Q\left(\gamma_{-}(a)\right)<-C a^{k} .
$$

Mostraremos que existe uma curva de mínimos vertical, com as propriedades que desejamos, tangente na origem a $\gamma_{-}$.

Seja $s$ a ordem do primeiro jato não identicamente nulo de $f$. É simples ver que $s \leq k, j^{s} f$ é um polinômio homogêneo de grau $s$ e uma das seguintes situações acontece:

1. $j^{s} f$ mostra que $f$ não tem mínimo na origem.

2. $j^{s} f$ tem mínimo estritamente brando em $\mathrm{O}$.

No primeiro caso, temos $s=k$ e o resultado que procuramos segue-se trivialmente. É imediato ver que a curva $\gamma_{-}$citada anteriormente é uma semi-reta que satisfaz as condições enunciadas. 
Concentraremo-nos então na situação em que $s<k$ e $j^{s} f$ é um polinômio homogêneo de grau $s$ com mínimo estritamente brando na origem.

Lembremos que $j^{s} f^{-1}\{0\}$ é um conjunto finito de retas todas passando pela origem.

Lema 5.1 Seja $\Lambda:[0 ; \varepsilon] \longrightarrow \mathrm{R}^{2}$ uma curva algébrica com $\Lambda(0)=$ $O$. Se $\Lambda$ é tangente na origem a uma semi-reta em que $j^{s} f$ não se anula, então a ordem de $P$ em $\Lambda$ és e $f \circ \Lambda$ tem mínimo estrito em 0 .

Prova: Seja $\ell$ a semi-reta à qual $\Lambda$ é tangente na origem. Façamos uma rotação que faça $\ell$ tornar-se o semi-eixo dos $x$.

Após esta rotação é claro que $j^{s} f(x ; 0)=a x^{s}$, com $a>0$.

Usando agora a parametrização canônica de $\Lambda$ segue-se imediatamente o resultado.

Uma conseqüência imediata deste fato é que a curva $\gamma_{-}$citada acima é tangente na origem a uma das semi-retas em que $j^{s} f$ se anula. Seja $\ell_{0}$ essa semi-reta.

Estamos agora em condições de terminar a prova da existência da curva de mínimos vertical neste caso.

Consideraremos a rotação que transforma $\ell_{0}$ no semi-eixo dos $x$ positivo e trabalharemos neste novo sistema de coordenadas.

Como $j^{s} f$ é um polinômio homogêneo não nulo, com mínimo estritamente brando na origem, e que se anula no semi-eixo $x \geq 0$, podemos escolher $\lambda>0$ tal que no cone

$$
C_{\lambda}=\left\{(x ; y) \in \mathrm{R}^{2}: x \geq 0,-\lambda x \leq y \leq \lambda x\right\}
$$

os únicos pontos em que $j^{s} f$ se anula sejam os da semi-reta $(x ; 0), x \geq$ 0 . 
Daqui e do fato 5.1 resulta que tanto $j^{k} f(x ; \lambda x), x \geq 0$ como $j^{k} f(x ;-\lambda x), x \geq 0$ possuem um mínimo de ordem $s$ na origem.

Por outro lado, como $\gamma_{-}$é tangente na origem ao semi-eixo dos $x$ positivos, tem-se que existe $\varepsilon_{0}>0$ tal que $\widetilde{\Gamma}(t) \in C_{\lambda}$ se $0<t<\varepsilon_{0}$.

Como $\widetilde{\Gamma}$ é algébrica e é transversal às esferas de centro na origem, resulta que essa curva também é trasversal às retas $x=$ $x_{0}$, para valores positivos de $x_{0}$ suficientemente pequenos.

Escrevendo $\widetilde{\Gamma}(t)=(x(t) ; y(t))$ vemos que, em $\left[0 ; \varepsilon_{0}[\right.$, a função $x(t)$ é estritamente crescente e tomando $\varrho=x\left(\varepsilon_{0}\right)$ vem que, para todo $\xi, 0<\xi<\varrho$, exite um único $t_{\xi} \in\left(0 ; \varepsilon_{0}\right)$ tal que $x_{1}\left(t_{\xi}\right)=\xi$ e existe $y_{x} i \in[-\lambda \xi ; \lambda \xi]$ tal que

$$
\min \left\{j^{k} f(\xi ; y):-\lambda \xi \leq y \leq \lambda \xi\right\}=f\left(\xi ; y_{\xi}\right) \leq j^{k} f(\widetilde{\Gamma})\left(t_{\xi}\right)<0 .
$$

Lembrando que $j^{k} f(x ;-\lambda x)$ e $j^{k} f(x ; \lambda x)$ têm mínimo estrito na origem, vem que $y_{\xi} \in(-\lambda \xi ; \lambda \xi)$.

Isto mostra que $\frac{\partial j^{k} f}{\partial y}\left(\xi ; y_{\xi}\right)=0$ e, portanto,

$$
A=\left\{(x ; y) \in \mathrm{R}^{2}: \frac{\partial j^{k} f}{\partial y}(x ; y)=0\right\}
$$

é um conjunto algébrico de dimensão 1 , do qual a origem não é um ponto isolado.

Assim, existe uma vizinhança $\Delta$ da origem, tal que $A \backslash\{O\} \cap \Delta$ é uma reunião finita de curvas algébricas todas aderentes à origem.

Ademais, vem de (5.1) que existe uma curva algébrica $\Gamma$ definida em $[0 ; \delta]$ tal que $\Gamma(0)=O, \Gamma(t) \in C_{\lambda} \cap A$, se $t>0$.

Sejam $\Gamma_{j}:\left[0 ; \delta_{j}\right] \longrightarrow \mathrm{R}^{2}, 1 \leq j \leq p$, as curvas algébricas que satisfazem as condições descritas no último parágrafo e vamos supor todas elas com a sua parametrização canônica. Tomemos $\delta_{0}=\min \delta_{j}: 1 \leq j \leq p$. 
Então, para $1 \leq j \leq p, \Gamma_{j}(t)=\left(t ; h_{j}(t)\right), 0 \leq t \leq \delta_{j}$, onde $h_{j}$ é uma função com série de potências de expoente fracionário.

Assim, fazendo $f_{j}=f \circ \Gamma_{j}$ existe $j_{0} \in\{1, \ldots, p\}$, tal que $f_{j_{0}}(x) \leq f_{j}(x)$, para todo $j$ e $0 \leq x \leq \delta_{0}$.

É claro que $\Gamma_{j_{0}}$ é a curva de mínimos desejada. 


\section{Bibliografia}

J [B ] Barone Netto, A., Jet-Detectable Extrema, Proc. Am. Math. Soc. vol. 92, no 4, pp 604-608, 1984.

[ BGZ ] Barone Netto, A., Gorni, G., Zampieri, G., Local Extrema of Analytic Functions, NoDEA - Nonlinear Diff. Equat. Appl. vol. 3, n⿳⺈ 3, pp 287-303, 1996.

[ C] Chetaev, N.G., On the Instability of Equilibrium in Some Cases where the Force Funcion is not Maximum (em russo), Prikl. Mat. Mech. vol 16, 89-93, 1952.

[D] Dumortier, F., Singularities of vector fields on the plane, J. Differential Equations vol.23, noำ 1, pp. 53-106, 1977.

[Gar-M.] Garcia, M.V.P. Estabilidade de Liapounof e k-decidibilidade, RT-MAP 9202, 30 pág., 1992.

[Gar-S.] Garcia, S.R.L. Energia Cinética e Estabilidade segundo Liapunov, Atas do 45o Seminário Brasileiro de Análise, Tomo II, pp. 769-773, Florianópolis, 1997.

[ H ] Hagedorn, P., Die Umkehrung der Stabilitätssätze von Lagrange-Dirichlet und Routh, Arch. Rat. Mech. Anal. vol.42, pp. 281-316, 1971. 
[ Koz - 1] Kozlov, V.V., A Conjecture on the Existence of Asymptotics Motions in Classical Mechanics, Funct. Anal. Appl. vol 17, 1983.

[ Koz - 2 ] Kozlov, V.V., Problemata Nova, ad quorum solutionem mathematici invitantur, Transl. of the Am. Math. Soc. (ser. 2) vol.168, n을, pp 141-171, 1995.

[ Kra] Krasovskii, N.N., Stability of Motion, Stanford University Press, Stanford, California, 1963.

[ Lal ] Laloy, M., On Equilibrium Instability for Conservative and Partially Dissipative Mechanical Systems, Sém. Math. Appl. Méca., Louvain, vol. 82, 1975.

[ LP ] Laloy, M., Peiffer, K., On the Instability Equilibrium when the Potential has a Non-Strict Local Minimum, Arch. Rat. Mech. Anal. vol. 78, no 3, pp. 213-222, 1980.

[ Liap ] Liapounof, A.M., Sur L'instabilité de l'équilibre dans certains cas où la fonction de forces nt'est pas um maximum, J. Math. Pures Appl., ser. V, 3, pp. 81-94, 1897.

[MN] Moauro, V., Negrini, P. On the Invertion of the LagrangeDirichlet Theorem, Diff. and Int. Equations vol. 2, pp. 471-478, 1989.

[ MMN ] Maffei, C., Moauro, V., Negrini, P. On the Invertion of the Lagrange-Dirichlet Theorem in a Case of Nonhomoheneous Potential, Diff. and Int. Equations vol. 4, no4, pp. 767-782, 1991.

[N] Negrini, P. On the Inversion of Lagrange-Dirichlet Theorem Res. Inst. Mat. e Est. vol. 2, no 2, pp. 83-114, 1995. 
[ Pai ] Painlevé, P., Sur la Stabilité de ltéquilibre, C. R. Acad. Sci. Paris (ser. A-b) vol. 138, pp 1555-1557, 1904.

[Pal-1] Palamodov, V.,Stability of Equilibrium in a Potential Field, Funct. Anal. Appl. vol. 11, pp. 277-289, 1978.

[Pal-2 ] Palamodov. V. Stability of Motion and Algebraic Geometry, Transl. of the Am. Math. Soc. (ser. 2) vol.168, no 25, pp 5-20, 1995.

[ RHL] Rouche, N., Habets, P., Laloy, M., Stability Theory by Liapunovts Direct Method, App. Math. Sci. vol. 22, SpringerVerlag, New York, 1997.

[RS ] Rumyantsev, V. V., Sosnitskiı, S. P., On the instability of the equilibrium of holonomic conservative systems, (Russian. Russian summary) Prikl. Mat. Mekh., vol. 57, no 6, pp. 144-166, 1993; (traduzido para o inglês) J. Appl. Math. Mech. vol.57, no 6, pp. 1101-1122 .1994.

[ Tag-1 ] Tagliaferro, S. An Inversion of the Lagrange-Dirichlet Stability Theorem, Arch. Rat. Mech. Anal. vol. 73, pp. 183-190, 1980.

[ Tag-2 ] Tagliaferro, S. Stability for two Dimensional Analytic Potentials, J. Diff. Equations vol. 35, pp. 248-265, 1980.

[ Tag-3 ] Tagliaferro, S. Instability of an Equilibrium in a Potential Field, Arch. Rat. Mech. Anal. vol. 109, 2, pp. 183-194, 1990.

[ Tak ] Takens, F., Singularities of vector fields, Inst. Hautes Études Sci. Publ. Math. vol. 43, pp. 47-100, 1974. 
[W] Walker, R.J., Algebraic Curves, 200 pág., Princeton Math. Series 13, Princeton Univ. Press, 1950. 